TRANSACTIONS OF THE

AMERICAN MATHEMATICAL SOCIETY

Volume 358, Number 5 , Pages 2011-2055

S 0002-9947(05)04016-X

Article electronically published on December 20, 2005

\title{
NEW PROPERTIES OF CONVEX FUNCTIONS IN THE HEISENBERG GROUP
}

\author{
NICOLA GAROFALO AND FEDERICO TOURNIER
}

\begin{abstract}
We prove some new properties of the weakly $H$-convex functions recently introduced by Danielli, Garofalo and Nhieu. As an interesting application of our results we prove a theorem of Busemann-Feller-Alexandrov type in the Heisenberg groups $\mathbb{H}^{n}, n=1,2$.
\end{abstract}

\section{INTRODUCTION}

Recently, there has been increasing interest in developing a theory of fully nonlinear sub-elliptic equations. The occurrence of such equations in CR geometry, as well as in stochastic control problems in financial mathematics (for this latter aspect see [Sto1], Sto2]), makes them an interesting object of study. Since the natural setting for these equations are graded nilpotent Lie groups, also known as Carnot groups, it is desirable to obtain an existence and regularity theory both local, and for boundary value problems, in such ambients.

With this objective in mind, we recall that a fundamental aspect of the classical theory of fully nonlinear equations is the connection between convexity and the Monge-Ampère equation

$$
\operatorname{det}\left(D^{2} u\right)=f(x, u, D u) .
$$

Such an equation is degenerate elliptic on $C^{2}$ convex functions, and elliptic on uniformly convex ones. In a Carnot group $\boldsymbol{G}$ there is an interesting class of equations related to (1.1), which we might say is of Monge-Ampère type. Let $X=\left\{X_{1}, \ldots, X_{m}\right\}$ be an orthonormal system of bracket-generating left-invariant vector fields. We recall here that if $\mathfrak{g}=V_{1} \oplus \ldots \oplus V_{r}$ represents a grading of the Lie algebra on $\boldsymbol{G}$, with $\left[V_{1}, V_{i}\right]=V_{i+1}, i=1, \ldots, r-1,\left[V_{1}, V_{r}\right]=\{0\}$, then $X$ is obtained by the formula $X_{i}(g)=\left(L_{g}\right)_{*}\left(e_{i}\right)$, where $\left\{e_{1}, \ldots, e_{m}\right\}$ is a fixed orthonormal basis of $V_{1}$, and $\left(L_{g}\right)_{*}$ denotes the differential of left-translations on $\boldsymbol{G}$. Given an open set $\Omega \subset \boldsymbol{G}$, we denote by $\Gamma^{k}(\Omega)$ the Folland-Stein space of functions having continuous

Received by the editors February 13, 2004.

2000 Mathematics Subject Classification. Primary 35H20; Secondary 26B25, 20F18.

Key words and phrases. Sub-elliptic fully nonlinear equations, monotonicity of Monge-Ampère measures, sub-elliptic cones, Busemann-Feller-Alexandrov theorem.

The first author was supported in part by NSF Grants No. DMS-0070492 and No. DMS0300477.

This work was presented by the first author in a Colloquium lecture at the University of Missouri, Columbia, in April 2003, and at the AMS Meeting at San Francisco State University in May 2003. 
derivatives up to order $k$ with respect to the vector fields $X_{1}, \ldots, X_{m}$. We emphasize that when the step of the group is $r=2$, then $\Gamma^{2}(\Omega) \subset C^{1}(\Omega)$. For a smooth function $u$, denote by $\operatorname{Hess}_{X}(u)=\left[u_{, i j}\right]$ its symmetrized horizontal Hessian, i.e., the $m \times m$ matrix with entries

$$
u_{, i j} \stackrel{\text { def }}{=} \frac{X_{i} X_{j} u+X_{j} X_{i} u}{2}
$$

The horizontal Monge-Ampère equation in $\boldsymbol{G}$ is defined by

$$
\operatorname{det} \operatorname{Hess}_{X}(u)=f(g, u, X u) \text {. }
$$

The geometric meaning of (1.3) is as follows. If we consider the horizontal subbundle $H \boldsymbol{G}=\bigcup_{g \in \boldsymbol{G}} H_{g} \subset T \boldsymbol{G}$, where $g \rightarrow H_{g}$ denotes the distribution of planes spanned by the system $X$, then the (Riemannian) Gaussian curvature $K_{H}$ in $g$ of the graph of the restriction of $u$ to $H_{g}$ is given by the equation

$$
\operatorname{det} \operatorname{Hess}_{X}(u)=K_{H}(g)\left(1+|X u|^{2}\right)^{(m+2) / 2}
$$

which is of the type (1.3). The question thus naturally arises of when such an equation, or more in general (1.3), is elliptic. In the paper DGN1 Danielli, Nhieu and the first author have introduced a geometric notion of convexity, called weak $H$-convexity. Among other things they proved that (1.3) is (degenerate) elliptic precisely on the class of $u \in \Gamma^{2}(\boldsymbol{G})$ which are weakly $H$-convex; see Theorem 2.2. This shows that, despite its many inherent difficulties, the notion of weak $H$-convexity is the appropriate one for studying (1.3).

In this paper we take up the investigations in DGN1 and develop some new properties of weakly $H$-convex functions in the Heisenberg group $\mathbb{H}^{n}$. Our main results are Theorems $2.1,6.8,7.1$, and 7.8 .

The Heisenberg group is the simplest prototype of a Carnot group of step two; see $[\mathrm{S}]$. It can be identified with the Euclidean space $\mathbb{C}^{n} \times \mathbb{R} \cong \mathbb{R}^{2 n+1}$, endowed with the nonabelian group law

$$
g \circ g^{\prime}=(x, y, t) \circ\left(x^{\prime}, y^{\prime}, t^{\prime}\right)=\left(x+x^{\prime}, y+y^{\prime}, t+t^{\prime}+\frac{1}{2}\left(\left\langle x, y^{\prime}\right\rangle-\left\langle x^{\prime}, y\right\rangle\right)\right) \text {. }
$$

The Heisenberg algebra admits the decomposition $\mathfrak{h}_{n}=V_{1} \oplus V_{2}$, where $V_{1}=\mathbb{R}^{2 n} \times$ $\{0\}_{t}$, and $V_{2}=\{0\}_{\mathbb{R}^{2 n}} \times \mathbb{R}_{t}$. Identifying $\mathfrak{h}_{n}$ with the space of left-invariant vector fields on $\mathbb{H}^{n}$, one easily recognizes that a basis for $\mathfrak{h}_{n}$ is given by the $2 n+1$ vector fields

$$
\left\{\begin{array}{l}
\left(L_{g}\right)_{*}\left(\frac{\partial}{\partial x_{i}}\right) \stackrel{\text { def }}{=} X_{i}=\frac{\partial}{\partial x_{i}}-\frac{y_{i}}{2} \frac{\partial}{\partial t} \\
\left(L_{g}\right)_{*}\left(\frac{\partial}{\partial y_{i}}\right) \stackrel{\text { def }}{=} X_{n+i}=\frac{\partial}{\partial y_{i}}+\frac{x_{i}}{2} \frac{\partial}{\partial t} \\
\left(L_{g}\right)_{*}\left(\frac{\partial}{\partial t}\right) \stackrel{\text { def }}{=} T=\frac{\partial}{\partial t},
\end{array}\right.
$$

and that the only nontrivial commutation relation is

$$
\left[X_{i}, X_{n+j}\right]=T \delta_{i j}, \quad i, j=1, \ldots, n
$$


Concerning Theorem 2.1 we mention that, while an earlier version of this paper was in progress, Gutierrez and Montanari announced the following result for the first Heisenberg group $\mathbb{H}^{1}$ (see Theorem 3.1 in [GM]).

Theorem 1.1. Let $\Omega \subset \mathbb{H}^{1}$ be a $C^{1}$ bounded open set, and let $u, v \in C^{2}(\bar{\Omega})$ be such that $u+v$ is a weakly $H$-convex function, $v<u$ in $\Omega$ and $v=u$ on $\partial \Omega$. Then

$$
\begin{aligned}
& \int_{\Omega}\left\{\operatorname{det} \operatorname{Hess}_{X}(u)+\frac{3}{4}(T u)^{2}\right\} d g \\
& \leq \int_{\Omega}\left\{\operatorname{det} \operatorname{Hess}_{X}(v)+\frac{3}{4}(T v)^{2}\right\} d g
\end{aligned}
$$

This result, like our Theorem 2.1, is inspired to the monotonicity theorem for convex functions first proved in a classical paper by Krylov [K], except that now one also has the $L^{2}$ norm of the commutator $T u$ in the integrand. At the time Theorem 1.1 was announced we had independently developed for the first Heisenberg group computations analogous to those in GM], but we were still uncertain about the role of the term $(T u)^{2}$ and felt that perhaps it should have been absorbed (i.e., controlled) by the term $\operatorname{det} \operatorname{Hess}_{X}(u)$. However, in a private conversation Gutierrez expressed to the first author his conviction that the term $(T u)^{2}$ should not be absorbed by det $\operatorname{Hess}_{X}(u)$. Following such a conversation it immediately occurred to us that Gutierrez's remark was in fact confirmed by a counterexample recently constructed in [DGN2]. This point is discussed in detail in Section 9, but see also Remark 6.7.

Our Theorem 2.1 represents a generalization of Theorem 1.1 to the Heisenberg group $\mathbb{H}^{2}$. However, our work differs from that in [GM] in three aspects. First of all, workers in the field traditionally accept as a given that any result which holds true for $\mathbb{H}^{1}$ easily extends to the higher-dimensional groups $\mathbb{H}^{n}$. This is not the case for Theorem 1.1. While the proof of the latter closely follows the original argument of Krylov $\mathrm{K}$ for the classical case, the proof of Theorem 2.1 is very involved and has entailed a substantial effort. For $\mathbb{H}^{2}$ the situation is incredibly more complex than that of $\mathbb{H}^{1}$, and to successfully handle the new Lagrangian terms involved has required several new ideas. We believe that our analysis of the Heisenberg group $\mathbb{H}^{2}$ can be profitably used as a paradigm of the situation for $\mathbb{H}^{n}$, with $n \geq 3$.

A second distinction between our work and the forthcoming paper [GM] is that in the latter paper the central motivation for proving Theorem 1.1 is to use it as the main tool in the proof of the following result (Theorem 5.5 in [GM]), which the authors call "a maximum principle similar to Alexandrov's estimate".

Theorem 1.2. Let $u \in C^{2}(B)$ be a weakly $H$-convex function in the gauge ball $B=B(0, R) \subset \mathbb{H}^{1}$, and suppose that $u=0$ on $\partial B$. For every $g_{o} \in B$ there exists a constant $C>0$, depending on $\operatorname{dist}\left(g_{o}, \partial B\right)$, such that

$$
\left|u\left(g_{o}\right)\right| \leq C\left(\int_{B}\left[\operatorname{det} \operatorname{Hess}_{X}(u)+\frac{3}{4}(T u)^{2}\right]\right)^{1 / 2} d g .
$$

We mention in passing that, although there is no explicit estimate on the constant $C$ in [GM], if one keeps track of the various cases one recognizes that it blows up at the boundary like a negative power of $\operatorname{dist}\left(g_{o}, \partial B\right)$. This is obviously not optimal since by assumption $u$ vanishes on $\partial B$, and thereby one should expect $C$ to vanish as a positive power of $\operatorname{dist}\left(g_{o}, \partial B\right)$. Establishing such correct dependence seems however a more difficult question. 
Theorem 1.2 is deduced by means of Theorem [1.1 various comparison theorems, and a lengthy analysis of the geometry of the gauge balls. Although this line of reasoning seems natural if one proceeds by analogy with Krylov's alternative approach to the classical geometric maximum principle of Alexandrov-BakelmanPucci in [K], it should be noted that Theorem 1.2 is not a maximum principle of geometric type. To clarify this point we present in Section 8 a completely elementary proof of such a result which does not use any of the tools in GM], and in particular makes no use of the monotonicity Theorem 1.1. In fact, for every $\mathbb{H}^{n}$ we prove a stronger statement, Theorem 8.3 , that can be directly deduced from the standard one-dimensional Poincaré inequality and a compactness result for weakly $H$-convex functions, which is Theorem 9.2 in DGN1.

The third difference between our paper and [GM] is that one of our main motivations for proving the monotonicity Theorem 2.1 was to use it to bridge the gap between the above-mentioned compactness Theorem 9.2 in [DGN1] and the integral version of the Busemann-Feller-Alexandrov due to Ambrosio and Magnani [AM]. This important aspect seems to have gone unnoticed in [GM]. Establishing a pointwise result of Busemann-Feller-Alexandrov type, i.e., the existence a.e. of the (nonsymmetrized) second derivatives of a weakly $H$-convex function was one of the main steps in the program set forth in DGN1]. In Theorem 7.8 of this paper we have been able to give an affirmative answer to this question for the first two Heisenberg groups. We also mention that in the forthcoming article [DGNT] we will study the Busemann-Feller-Alexandrov theorem in the general setting of Carnot groups of step two.

We now describe the results in this paper. For a function $u$ on $\mathbb{H}^{2}$ we consider the $4 \times 4$ matrix $\operatorname{Hess}_{X}(u)=\left[u_{i j}\right]_{i, j=1, \ldots, 4}$ defined by (1.2). We note that in $\mathbb{H}^{2}$ a basis of the Lie algebra of left-invariant vector fields is given by $\left\{X_{1}, X_{2}, X_{3}, X_{4}, T\right\}$, where the latter are defined by (1.4). We note explicitly that we now have from (1.5) the following nontrivial commutation relations

$$
\left[X_{1}, X_{3}\right]=\left[X_{2}, X_{4}\right]=T
$$

all other commutators being trivial. For a function $u \in \Gamma^{2}\left(\mathbb{H}^{2}\right)$ we introduce the fully nonlinear operator acting on $u$ as follows:

$$
\begin{aligned}
\mathcal{S}_{m a}(u) & =\operatorname{det} \operatorname{Hess}_{X}(u)+\frac{3}{4}\left\{\operatorname{det}\left(\begin{array}{ll}
u, 11 & u_{, 13} \\
u_{, 13} & u_{, 33}
\end{array}\right)+\operatorname{det}\left(\begin{array}{ll}
u_{, 22} & u_{, 24} \\
u_{, 24} & u_{, 44}
\end{array}\right)\right. \\
& \left.+2 \operatorname{det}\left(\begin{array}{ll}
u_{, 12} & u_{, 14} \\
u_{, 23} & u_{, 34}
\end{array}\right)\right\}(T u)^{2}+\frac{5}{16}(T u)^{4} .
\end{aligned}
$$

We call such an operator the generalized sub-elliptic Monge-Ampère operator. The adjective sub-elliptic is well justified by the appearance of the terms containing the commutator $T u$.

In Section 2 we prove Theorem 2.1. As we have already mentioned, one important consequence of the basic identity (2.6) in Theorem 2.1 is that if $u$ and $v$ are (sufficiently smooth) weakly $H$-convex functions in a domain $\Omega \subset \mathbb{H}^{2}, u \geq v$ in $\Omega$, and $u=v$ on $\partial \Omega$, then

$$
\int_{\Omega} S_{m a}(u) d g \leq \int_{\Omega} S_{m a}(v) d g
$$


For functions on $\mathbb{H}^{1}$ such a fully nonlinear operator takes the simpler form

$$
S_{m a}(u)=\operatorname{det} \operatorname{Hess}_{X}(u)+\frac{3}{4}(T u)^{2}
$$

and therefore (1.8) says nothing new with respect to Theorem 1.1.

In Section 3 we prove that, remarkably, on every smooth weakly $H$-convex function in $\mathbb{H}^{2}$ the fully nonlinear operator in (1.7) is positive, i.e., $\mathcal{S}_{m a}(u) \geq 0$. While the positivity of $\operatorname{det} \operatorname{Hess}_{X}(u)$ is guaranteed by Theorem 2.2. because of the $2 \times 2$ minors within curly brackets in the definition of $\mathcal{S}_{m a}(u)$ such important property is a priori very much in doubt. On the other hand, such positivity plays a crucial role in the applications; see for instance the proof of Theorem 7.1 .

In Section 4 we generalize Theorem 1.1 to the four-dimensional Engel group of step $r=3$. Such a group is interesting since it constitutes a higher step model of the Heisenberg group. Because the nonvanishing commutators are of order three, Theorem 4.1 displays a new feature with respect to the case of the Heisenberg group. In this connection, we mention the article DGNT, which contains an interesting generalization of Theorem 4.1 to arbitrary Carnot groups.

In Section [5 we consider the sub-elliptic "cones" proposed in [DGN1], and establish a basic comparison result between weakly $H$-convex functions and some appropriate regularizations of such cones; see Theorem 5.9. Here, we rely, among other things, on the comparison theorem of Bieske [B] for the $\infty$-sub-Laplacian; see also the recent generalization of this result due to C. Wang [Wa2].

In Section 6 we study the action of the fully nonlinear operator appearing in Theorem 2.1 on the regularized sub-elliptic cones $\Gamma_{H, \epsilon}$ in (5.3). The main result is Theorem 6.8, which describes the limit as $\epsilon \rightarrow 0$ of the generalized Monge-Ampère measure of $\Gamma_{H, \epsilon}$. It is important to stress here that, although the sub-elliptic cone does constitute an explicit singular solution of the horizontal Monge-Ampère equation (1.3) with zero right-hand side (see Theorem 10.9 in DGN1]), it is different from the classical case in that it is not a fundamental solution of the latter; see Proposition 6.6. However, Theorem 6.8 states that the cone is a "fundamental solution" of the generalized Monge-Ampère operator appearing in Theorem 2.1 . We emphasize that Proposition 6.6 also shows that the analogue of the AlexandrovBakelman-Pucci (ABP) estimate (1.9) below cannot possibly hold. As we explain in Section 9 this negative phenomenon also follows from the results in DGN2.

In Section 7 we take up a beautiful idea in the paper by Trudinger and Wang TW, combined with the results in Sections 2, 5] and [6, to derive some basic consequences of Theorem 2.1 and of Theorem 6.8. One of them is the local estimate of the generalized Monge-Ampère measure from above; see Theorem 7.1, Such an estimate, which should be thought of as a fully nonlinear Caccioppoli-type inequality, proves in particular that the commutator of every weakly $H$-convex function belongs to $L_{l o c}^{2}$, hence the function itself belongs to the space $B V_{X, l o c}^{2}$, i.e., all its second derivatives along the vector fields (not just the symmetrized ones) are Radon measures. This fundamental information allows us to close the gap between the basic $L^{\infty}-L^{1}$ compactness estimates in Theorem 9.2 from DGN1 and the integral version of the Busemann-Feller-Alexandrov theorem due to Ambrosio and Magnani [AM]. We thus obtain a pointwise version for weakly $H$-convex functions of the theorem of Busemann-Feller and Alexandrov on the existence a.e. of the second derivatives of a convex function; see Theorem 7.8 , 
In Section 8 we give a simple proof of a global estimate from below for the generalized Monge-Ampère measure; see Corollary 8.4. As we have already mentioned, despite their resemblance, there exists a marked discrepancy between such a result and the geometric Alexandrov-Bakelman-Pucci estimate

$$
\sup _{\Omega}|u| \leq \frac{\operatorname{diam}(\Omega)}{\omega_{n}^{1 / n}}\left(\int_{\Omega}\left|\operatorname{det} D^{2} u\right| d x\right)^{1 / n},
$$

valid in a bounded domain $\Omega \subset \mathbb{R}^{n}$ for any convex function $u \in C^{2}(\Omega) \cap C(\bar{\Omega})$, such that $u=0$ on $\partial \Omega$.

In Section 9 we connect the already-mentioned negative phenomenon in Remark 6.7 to certain conjectured a priori inequalities of ABP type which presently constitute a fundamental open question to further the development of the theory of fully nonlinear sub-elliptic equations.

\section{Monotonicity For SMOOTh CONVEX FUnCtions IN THE HEISENBERG GROUPS}

In $\mathrm{K}$ Krylov proved the following result (in fact, he proved a parabolic version of it). Consider a $C^{1}$ bounded open set $\Omega \subset \mathbb{R}^{n}$, and let $u, v \in C^{3}(\bar{\Omega})$ be convex functions such that $u \geq v$ in $\Omega$ and $u=v$ on $\partial \Omega$; then

$$
\int_{\Omega} \operatorname{det}\left(D^{2} u\right) d x \leq \int_{\Omega} \operatorname{det}\left(D^{2} v\right) d x .
$$

The aim of this section is to establish Theorem 2.1. The latter provides a basic sub-elliptic version of (2.1), and represents a fundamental property of weakly $H$ convex functions. For the first Heisenberg group $\mathbb{H}^{1}$, such a monotonicity result can be given an elementary proof; see Theorem 1.1 in GM]. The case of $\mathbb{H}^{2}$ involves a large amount of additional work and new ideas with respect to the classical case, as one needs to exploit to the fullest extent the intrinsic symmetries of the Heisenberg group in order to handle the complex integrands involved.

For a $C^{1}$ domain $\Omega$ in a Carnot group $\boldsymbol{G}$ we denote by $\nu$ the Riemannian outer unit normal to $\partial \Omega$. We introduce the horizontal normal to $\partial \Omega$ (see [DGN3])

$$
\nu_{X}=\left(\nu_{X, 1}, \ldots, \nu_{X, m}\right)^{T},
$$

whose components are defined by

$$
\nu_{X, i}=\left\langle X_{i}, \nu\right\rangle
$$

When the group $\boldsymbol{G}$ is $\mathbb{H}^{n}$, we have $\nu_{X}=\left(\nu_{X, 1}, \ldots, \nu_{X, 2 n}\right)^{T}$. In such a case, we will indicate by $\nu_{X}^{\perp}=J\left(\nu_{X}\right)$ the image of $\nu_{X}$ through the symplectic $2 n \times 2 n$ matrix

$$
J=\left(\begin{array}{cc}
0 & I \\
-I & 0
\end{array}\right),
$$

where 0 and $I$ respectively indicate the zero and the identity matrix in $\mathbb{R}^{n}$. Thus, when for instance $n=2$, we have

$$
\nu_{X}^{\perp}=\left(\nu_{X, 3}, \nu_{X, 4},-\nu_{X, 1},-\nu_{X, 2}\right)^{T} .
$$

Henceforth in this paper, we adopt the summation convention over repeated indices. Also for ease of notation, given a function $u \in \Gamma^{2}(\Omega)$, where $\Omega$ is an open subset of a Carnot group, we let

$$
H(u)=\operatorname{det} \operatorname{Hess}_{X}(u) .
$$


Theorem 2.1. Let $\Omega \subset \mathbb{H}^{2}$ be a $C^{1}$ bounded open set, and consider two weakly $H$-convex functions $u, v \in \Gamma^{3}(\bar{\Omega})$ such that $u \geq v$ in $\Omega$ and $u=v$ on $\partial \Omega$. For $0 \leq s \leq 1$ we set

$$
z=z(g, s) \stackrel{\text { def }}{=}(1-s) u(g)+s v(g), \quad g \in \Omega .
$$

Denoting by $\mathcal{S}_{m a}(z)$ the modified sub-elliptic Monge-Ampère operator (1.7) acting on the function $g \rightarrow z(g, s)$, we have

$$
\begin{aligned}
& \frac{d}{d s} \int_{\Omega} \mathcal{S}_{m a}(z) d g \\
& =\int_{\partial \Omega} \frac{\partial H(z)}{\partial z_{, i j}} \nu_{X, i} \nu_{X, j}\left|\nabla z_{s}\right| d \sigma+\frac{3}{4} \int_{\partial \Omega}\left\langle H e s s_{X}(z) \nu_{X}^{\perp}, \nu_{X}^{\perp}\right\rangle(T z)^{2}\left|\nabla z_{s}\right| d \sigma .
\end{aligned}
$$

Since $u$ and $v$ are weakly $H$-convex, $z$ is also weakly $H$-convex, and Theorem 2.2 below guarantees that the right-hand side of (2.6) is nonnegative. We thus conclude that the function $s \longrightarrow \int_{\Omega} \mathcal{S}_{m a}(z(g, s)) d g$ is increasing on $[0,1]$, hence in particular

$$
\int_{\Omega} \mathcal{S}_{m a}(u) d g \leq \int_{\Omega} \mathcal{S}_{m a}(v) d g .
$$

To prepare for the proof of Theorem 2.1 we begin by recalling the relevant notion of convexity introduced in DGN1. Given a Carnot group $\boldsymbol{G}$, a function $u: \boldsymbol{G} \rightarrow \mathbb{R}$ is called weakly $H$-convex if for every $g \in \boldsymbol{G}$ and $0 \leq \lambda \leq 1$ one has

$$
u\left(g \delta_{\lambda}\left(g^{-1} g^{\prime}\right)\right) \leq(1-\lambda) u(g)+\lambda u\left(g^{\prime}\right), \quad \text { for every } g^{\prime} \in H_{g},
$$

where $H_{g}$ indicates the horizontal plane through $g \in \boldsymbol{G}$. In (2.8) we have indicated by $\delta_{\lambda}: \boldsymbol{G} \rightarrow \boldsymbol{G}$ the anisotropic dilations on $\boldsymbol{G}$. The point $g \delta_{\lambda}\left(g^{-1} g^{\prime}\right)$ denotes the twisted convex combination of $g$ and $g^{\prime}$ based at $g$. We also mention the paper [LMS, where the authors have introduced for the Heisenberg group $\mathbb{H}^{n}$ a notion of convexity in the viscosity sense of [CIL, called $v$-convexity, and derived various properties for the relevant convex functions. While it is easy to see that every weakly $H$-convex function is also $v$-convex, the more delicate reverse implication has been recently established in the papers [BR], Wa3], [M]. As a consequence, one now knows that the geometric notion of weak $H$-convexity is in fact equivalent to that of $v$-convexity.

In the abelian case, when the step of the grading of $\mathfrak{g}$ is simply $r=1$, we can identify $V_{1}$ with $\mathbb{R}^{m}$, and then $X_{i}=\partial / \partial x_{i}$ is just its standard basis. In this situation, for every $g \in G \cong \mathbb{R}^{m}$ the horizontal plane $H_{g}$ can be identified with $\mathbb{R}^{m}$ itself, and thus the notion of weak $H$-convexity 2.8 gives back the classical notion of convexity. In the nonabelian case $r>1$, however, things are drastically different, and the notion of weak $H$-convexity turns out to be much harder to work with than its classical predecessor since: 1) It lacks symmetry, in the sense that in (2.8) the base point $g$ plays a privileged role, and it is not possible to interchange $g$ and $\left.g^{\prime} ; 2\right)$ At every point $g \in \boldsymbol{G}$ it only guarantees a quantitative control of the function $u$ on the lower-dimensional manifold $H_{g}$. Obtaining control on a set of full measure from such information is a very hard task. As a consequence, the theory of weakly $H$-convex functions displays many new challenging aspects.

Despite these unsettling obstructions, however, the notion of weak $H$-convexity turns out to be the correct one for studying (1.3). In fact, thanks to the following result, which is Theorem 5.11 in [DGN1, but see also [LMS] for a similar result 
for the Heisenberg group, the equation (1.3) is elliptic precisely on those functions $u \in \Gamma^{2}(\boldsymbol{G})$ which are uniformly weakly $H$-convex.

Theorem 2.2. A function $u \in \Gamma^{2}(\boldsymbol{G})$ is (uniformly) weakly $H$-convex if and only if $\operatorname{Hess}_{X}(u)$ is (definite) semi-definite positive on $\boldsymbol{G}$.

We now turn to the main objective of this section. We start with a calculus lemma for arbitrary Carnot groups inspired to Krylov's approach in $[\mathrm{K}]$.

Lemma 2.3. Let $\boldsymbol{G}$ be a Carnot group and $\Omega \subset \boldsymbol{G}$ be a $C^{1}$ bounded open set. Consider two functions $u, v \in \Gamma^{3}(\bar{\Omega}) \cap C^{1}(\bar{\Omega})$ such that $u \geq v$ in $\Omega$ and $u=v$ on $\partial \Omega$. For $0 \leq s \leq 1$ we set

$$
z=z(g, s) \stackrel{\text { def }}{=}(1-s) u(g)+s v(g), \quad g \in \Omega,
$$

and

$$
f(s)=\int_{\Omega} H(z(g, s)) d g, \quad 0 \leq s \leq 1
$$

We have

$$
f^{\prime}(s)=\int_{\partial \Omega} \sum_{i, j=1}^{m} \frac{\partial H(z)}{\partial z_{, i j}} \nu_{X, i} \nu_{X, j}\left|\nabla z_{s}\right| d \sigma-\int_{\Omega} \sum_{i, j=1}^{m} X_{i} \frac{\partial H(z)}{\partial z_{, i j}} X_{j}\left(z_{s}\right) d g
$$

where we have indicated with $d \sigma$ the Riemannian volume measure on $\partial \Omega$.

Proof. In the sequel we will indicate with $z_{s}$ the partial derivative

$$
\frac{\partial z}{\partial s}=v-u
$$

We note explicitly that $z_{s} \leq 0$ in $\Omega$, and $z_{s}=0$ on $\partial \Omega$. Since by the $C^{1}$ assumption on $u$ and $v$ we also have $z \in C^{1}(\bar{\Omega})$, the Riemannian unit normal $\nu$ to $\partial \Omega$ satisfies the relation

$$
\nabla z_{s}=\nu\left|\nabla z_{s}\right|
$$

where $\nabla$ indicates the Riemannian gradient in $\boldsymbol{G}$. A differentiation now gives

$$
f^{\prime}(s)=\int_{\Omega} \frac{\partial H(z)}{\partial z_{, i j}} \frac{\partial z_{, i j}}{\partial s} d g=\int_{\Omega} \frac{\partial H(z)}{\partial z_{, i j}}\left(z_{s}\right)_{, i j} d g .
$$

Using the definition (1.2), and integrating by parts, we find

$$
\begin{aligned}
f^{\prime}(s) & =\frac{1}{2} \int_{\partial \Omega} \frac{\partial H(z)}{\partial z_{, i j}} X_{j}\left(z_{s}\right)\left\langle X_{i}, \nu\right\rangle d \sigma-\frac{1}{2} \int_{\Omega} X_{i} \frac{\partial H(z)}{\partial z_{, i j}} X_{j}\left(z_{s}\right) d g \\
& +\frac{1}{2} \int_{\partial \Omega} \frac{\partial H(z)}{\partial z_{, i j}} X_{i}\left(z_{s}\right)\left\langle X_{j}, \nu\right\rangle d \sigma-\frac{1}{2} \int_{\Omega} X_{j} \frac{\partial H(z)}{\partial z_{, i j}} X_{i}\left(z_{s}\right) d g .
\end{aligned}
$$

Using (2.12) we see that

$$
X_{i}\left(z_{s}\right)=\left\langle X_{i}, \nu\right\rangle\left|\nabla z_{s}\right|=\nu_{X, i}\left|\nabla z_{s}\right| .
$$

Substitution in (2.13) gives

$$
f^{\prime}(s)=\int_{\partial \Omega} \frac{\partial H(z)}{\partial z_{, i j}} \nu_{X, i} \nu_{X, j}\left|\nabla z_{s}\right| d \sigma-\int_{\Omega} X_{i} \frac{\partial H(z)}{\partial z_{, i j}} X_{j}\left(z_{s}\right) d g,
$$

which is (2.11). 
Lemma 2.3 brings us to the essential new aspect of our study. We stress that in the abelian setting, i.e., when the group $\boldsymbol{G}$ is just Euclidean $\mathbb{R}^{m}$, then $\left\{X_{1}, \ldots, X_{m}\right\}$ is just the standard basis $\left\{\partial / \partial x_{1}, \ldots, \partial / \partial x_{m}\right\}$ of $\mathbb{R}^{m}$, and with $z_{i j}=\partial^{2} z / \partial x_{i} \partial x_{j}$ we have

$$
X_{i} \frac{\partial H(z)}{\partial z_{, i j}}=\sum_{i=1}^{m} \frac{\partial}{\partial x_{i}} \frac{\partial\left(\operatorname{det}\left(D^{2} z\right)\right)}{\partial z_{i j}} .
$$

Therefore, combining Lemma 2.3 with the following null-Lagrangian property of the determinant of the Hessian of a $C^{3}$ function (see Theorem 2 on p. 441 of $[\mathrm{E}$, or also $[\mathrm{Da}]$,

$$
\sum_{i=1}^{n} \frac{\partial}{\partial x_{i}} \frac{\partial\left(\operatorname{det}\left(D^{2} z\right)\right)}{\partial z_{i j}}=0, \quad j=1, \ldots, n,
$$

one immediately obtains from (2.11) the monotonicity of the functional

$$
E(z)(s)=\int_{\Omega} \operatorname{det} D^{2} z(x, s) d x
$$

which was established in $[\mathrm{K}$ in the proof of (2.1).

The remaning part of this section is devoted to finding, at least for the setting of the Heisenberg group $\mathbb{H}^{2}$, the appropriate replacement of the null-Lagrangian property (2.15), with the intent of proving Theorem 2.1. This turns out to be a highly nontrivial task. Instead of looking for a pointwise identity, we have derived a new integral version of (2.15).

We begin by analyzing, for a function $z \in \Gamma^{3}\left(\mathbb{H}^{2}\right)$, the special symmetries of the quantity

$$
\sum_{i=1}^{4} X_{i} \frac{\partial H(z)}{\partial z_{, i j}}, \quad j=1, \ldots, 4 .
$$

The next lemma describes the structure of this quantity.

Lemma 2.4. Let $z \in \Gamma^{3}\left(\mathbb{H}^{2}\right)$. There exist nonlinear functions of the symmetrized horizontal derivatives $z_{, i j}, A_{i}^{j} \in \Gamma^{1}\left(\mathbb{H}^{2}\right)$, such that for every $j=1, \ldots, 4$

$$
\sum_{i=1}^{4} X_{i} \frac{\partial H(z)}{\partial z_{, i j}}=\frac{3}{2} \sum_{\substack{i=1, \ldots, 4 \\ i \neq j}} A_{i}^{j} X_{i}(T z)
$$

Proof. The symmetrized horizontal Hessian of $z$ is given by

$$
\operatorname{Hess}_{X}(z)=\left(\begin{array}{cccc}
z_{, 11} & z_{, 12} & z_{, 13} & z_{, 14} \\
z_{, 21} & z_{, 22} & z_{, 23} & z_{, 24} \\
z_{, 31} & z_{, 32} & z_{, 33} & z_{, 34} \\
z_{, 41} & z_{, 42} & z_{, 43} & z_{, 44}
\end{array}\right) \text {. }
$$

From the cofactor expansion of the determinant of (2.18) we obtain for $j=$ $1, \ldots, 4$,

$$
H(z)=z_{, 1 j} C_{1 j}+\ldots+z_{, 4 j} C_{4 j}
$$

where we have denoted with $C_{i j}$ the cofactor of the element $z_{, i j}$ of the matrix $\operatorname{Hess}_{X}(z)$. This gives

$$
\frac{\partial H(z)}{\partial z_{, i j}}=C_{i j}
$$


We now analyze the case in which $j=1$ in (2.16). Using (2.19) we find that

$$
\begin{aligned}
\sum_{i=1}^{4} X_{i} \frac{\partial H(z)}{\partial z_{, i 1}}= & \sum_{i=1}^{4} X_{i} C_{i 1} \\
= & X_{1} \operatorname{det}\left(\begin{array}{lll}
z_{, 22} & z_{, 23} & z_{, 24} \\
z_{, 32} & z_{, 33} & z_{, 34} \\
z_{, 42} & z_{, 43} & z_{, 44}
\end{array}\right)-X_{2} \operatorname{det}\left(\begin{array}{lll}
z, 12 & z_{, 13} & z_{, 14} \\
z_{, 32} & z_{, 33} & z_{, 34} \\
z_{, 42} & z_{, 43} & z_{, 44}
\end{array}\right) \\
& +X_{3} \operatorname{det}\left(\begin{array}{lll}
z_{, 12} & z_{, 13} & z_{, 14} \\
z_{, 22} & z_{, 23} & z_{, 24} \\
z_{, 42} & z_{, 43} & z_{, 44}
\end{array}\right)-X_{4} \operatorname{det}\left(\begin{array}{lll}
z_{, 12} & z_{, 13} & z_{, 14} \\
z_{, 22} & z_{, 23} & z_{, 24} \\
z_{, 32} & z_{, 33} & z_{, 34}
\end{array}\right)
\end{aligned}
$$

For $i, j, k=1, \ldots, 4$, we now denote by $\{i j k\}$ the complex of all terms in the expansion (2.20) containing any of the derivatives $X_{i} z_{, j k}, X_{j} z_{, i k}, X_{k} z_{, i j}$. For instance, we have

$$
\{122\}=X_{1} z_{, 22} \operatorname{det}\left(\begin{array}{cc}
z, 33 & z, 34 \\
z, 43 & z, 44
\end{array}\right)-X_{2} z_{, 12} \operatorname{det}\left(\begin{array}{cc}
z, 33 & z, 34 \\
z, 43 & z, 44
\end{array}\right) .
$$

Likewise, we have

$$
\begin{aligned}
\{124\} & =X_{1} z_{, 24} \operatorname{det}\left(\begin{array}{ll}
z_{, 32} & z_{, 33} \\
z_{, 42} & z_{, 43}
\end{array}\right)+X_{1} z_{, 42} \operatorname{det}\left(\begin{array}{ll}
z_{, 23} & z_{, 24} \\
z_{, 33} & z_{, 34}
\end{array}\right) \\
& -X_{2} z_{, 14} \operatorname{det}\left(\begin{array}{cc}
z_{, 32} & z_{, 33} \\
z_{, 42} & z_{, 43}
\end{array}\right)-X_{4} z_{, 12} \operatorname{det}\left(\begin{array}{ll}
z_{, 23} & z_{, 24} \\
z_{, 33} & z_{, 34}
\end{array}\right),
\end{aligned}
$$

or also

$$
\{144\}=X_{1} z_{, 44} \operatorname{det}\left(\begin{array}{cc}
z_{, 22} & z_{, 23} \\
z, 32 & z_{, 33}
\end{array}\right)-X_{4} z_{, 14} \operatorname{det}\left(\begin{array}{ll}
z_{, 22} & z_{, 23} \\
z_{, 32} & z_{, 33}
\end{array}\right) .
$$

We now observe that, thanks to the commutation relations (1.6), one easily recognizes from the definition (1.2) of the symmetric derivatives that

$$
X_{1} z_{, 22}-X_{2} z_{, 12}=0, X_{1} z_{, 44}-X_{4} z_{, 14}=0,
$$

and so both the terms $\{122\}$ and $\{144\}$ vanish. As for $\{124\}$ we have from (1.6)

$$
X_{1} z_{, 24}-X_{2} z_{, 14}=-\frac{1}{2} X_{1}(T z), \quad X_{1} z_{, 42}-X_{4} z, 12=\frac{1}{2} X_{1}(T z),
$$

and so we also have $\{124\}=0$.

The first nonzero term in (2.20) is

$$
\begin{aligned}
\{123\}= & -X_{1} z_{, 23} \operatorname{det}\left(\begin{array}{cc}
z_{, 32} & z_{, 34} \\
z_{, 42} & z_{, 44}
\end{array}\right)-X_{1} z_{, 32} \operatorname{det}\left(\begin{array}{ll}
z_{, 23} & z_{, 24} \\
z_{, 43} & z_{, 44}
\end{array}\right) \\
& +X_{2} z_{, 13} \operatorname{det}\left(\begin{array}{ll}
z_{, 32} & z_{, 34} \\
z_{, 42} & z_{, 44}
\end{array}\right)+X_{3} z_{, 12} \operatorname{det}\left(\begin{array}{cc}
z_{, 23} & z_{, 24} \\
z_{, 43} & z_{, 44}
\end{array}\right) \\
& =\operatorname{det}\left(\begin{array}{cc}
z_{, 23} & z_{, 24} \\
z_{, 34} & z_{, 44}
\end{array}\right)\left\{\left(X_{2} z_{, 13}-X_{1} z_{, 23}\right)+\left(X_{3} z_{, 12}-X_{1} z_{, 32}\right)\right\} .
\end{aligned}
$$

A computation based on (1.6) now gives

$$
X_{2} z_{, 13}-X_{1} z, 23=-\frac{1}{2} X_{2}(T z), \quad X_{3} z_{, 12}-X_{1} z, 32=-X_{2}(T z) .
$$


Substituting in (2.21) we find

$$
\{123\}=-\frac{3}{2} X_{2}(T z) \quad \operatorname{det}\left(\begin{array}{ll}
z, 23 & z, 24 \\
z, 34 & z, 44
\end{array}\right)
$$

After similar computations we find for the other nonvanishing terms in (2.20) that

$$
\begin{aligned}
& \{133\}=\frac{3}{2} X_{3}(T z) \operatorname{det}\left(\begin{array}{cc}
z, 22 & z_{24} \\
z_{, 24} & z_{, 44}
\end{array}\right), \\
& \{134\}=-\frac{3}{2} X_{4}(T z) \quad \operatorname{det}\left(\begin{array}{cc}
z, 22 & z, 23 \\
z, 24 & z, 34
\end{array}\right) \text {, } \\
& \{234\}=\frac{1}{2} X_{3}(T z)\left\{\operatorname{det}\left(\begin{array}{ll}
z_{, 12} & z, 13 \\
z_{, 24} & z_{, 34}
\end{array}\right)+2 \operatorname{det}\left(\begin{array}{ll}
z_{, 12} & z_{, 14} \\
z_{, 23} & z_{, 34}
\end{array}\right)+\operatorname{det}\left(\begin{array}{ll}
z_{, 13} & z_{, 14} \\
z_{, 23} & z_{, 24}
\end{array}\right)\right\} \\
& =\frac{3}{2} X_{3}(T z) \quad \operatorname{det}\left(\begin{array}{cc}
z_{, 12} & z_{, 14} \\
z, 23 & z_{, 34}
\end{array}\right) \text {, } \\
& \{224\}=-\frac{3}{2} X_{2}(T z) \quad \operatorname{det}\left(\begin{array}{cc}
z, 13 & z, 14 \\
z, 33 & z, 34
\end{array}\right) \text {, } \\
& \{244\}=-\frac{3}{2} X_{4}(T z) \quad \operatorname{det}\left(\begin{array}{cc}
z, 12 & z, 13 \\
z, 23 & z, 33
\end{array}\right) \text {. }
\end{aligned}
$$

Inserting (2.22) and (2.23) into (2.20), we conclude that

$$
\sum_{i=1}^{4} X_{i} \frac{\partial H(z)}{\partial z, i 1}=\frac{3}{2}\left\{A_{2}^{1} X_{2}(T z)+A_{3}^{1} X_{3}(T z)+A_{4}^{1} X_{4}(T z)\right\}
$$

where the coefficients $A_{i}^{j}$ are appropriate nonlinear terms involving sums of $2 \times 2$ minors of the matrix $\operatorname{Hess}_{X}(z)$ which are easily computed from (2.22) and (2.23). This proves (2.16) when $j=1$. The remaining cases $j=2,3,4$ are proved similarly.

Since in the sequel it will be crucial to know the fully nonlinear operators $A_{i}^{j}$, we have collected their explicit expressions in the next lemma.

Lemma 2.5. In $\mathbb{H}^{2}$ the twelve fully nonlinear operators in Lemma 2.4 are given by the following formulas:

$$
\begin{aligned}
& A_{2}^{1}=-\operatorname{det}\left(\begin{array}{ll}
z_{, 23} & z_{, 24} \\
z_{, 34} & z_{, 44}
\end{array}\right)-\operatorname{det}\left(\begin{array}{ll}
z_{, 13} & z_{, 14} \\
z_{, 33} & z_{, 34}
\end{array}\right), \\
& A_{3}^{1}=\operatorname{det}\left(\begin{array}{ll}
z_{, 22} & z_{, 24} \\
z_{, 24} & z_{, 44}
\end{array}\right)+\operatorname{det}\left(\begin{array}{ll}
z_{, 12} & z_{, 14} \\
z_{, 23} & z_{, 34}
\end{array}\right), \\
& A_{4}^{1}=-\operatorname{det}\left(\begin{array}{ll}
z_{, 22} & z_{, 23} \\
z_{, 24} & z_{, 34}
\end{array}\right)-\operatorname{det}\left(\begin{array}{ll}
z_{, 12} & z_{, 13} \\
z_{, 23} & z_{, 33}
\end{array}\right) .
\end{aligned}
$$




$$
\begin{aligned}
& A_{1}^{2}=\operatorname{det}\left(\begin{array}{ll}
z, 13 & z_{, 14} \\
z_{, 33} & z_{, 34}
\end{array}\right)+\operatorname{det}\left(\begin{array}{ll}
z_{, 23} & z_{, 24} \\
z_{, 34} & z_{, 44}
\end{array}\right), \\
& A_{3}^{2}=-\operatorname{det}\left(\begin{array}{ll}
z_{, 12} & z_{, 24} \\
z_{, 14} & z_{, 44}
\end{array}\right)-\operatorname{det}\left(\begin{array}{ll}
z_{, 11} & z_{, 13} \\
z_{, 14} & z_{, 34}
\end{array}\right), \\
& A_{4}^{2}=\operatorname{det}\left(\begin{array}{ll}
z_{, 12} & z_{, 14} \\
z_{, 23} & z_{, 34}
\end{array}\right)+\operatorname{det}\left(\begin{array}{ll}
z_{, 11} & z_{, 13} \\
z_{, 13} & z_{, 33}
\end{array}\right) .
\end{aligned}
$$

$$
\begin{aligned}
& A_{1}^{3}=\operatorname{det}\left(\begin{array}{ll}
z_{, 14} & z_{, 12} \\
z_{, 34} & z_{, 23}
\end{array}\right)-\operatorname{det}\left(\begin{array}{ll}
z_{, 22} & z_{, 24} \\
z_{, 24} & z_{, 44}
\end{array}\right), \\
& A_{2}^{3}=\operatorname{det}\left(\begin{array}{ll}
z_{, 12} & z_{, 14} \\
z_{, 24} & z_{, 44}
\end{array}\right)+\operatorname{det}\left(\begin{array}{ll}
z_{, 11} & z_{, 14} \\
z_{, 13} & z_{, 34}
\end{array}\right), \\
& A_{4}^{3}=-\operatorname{det}\left(\begin{array}{ll}
z_{, 12} & z_{, 14} \\
z_{, 22} & z_{, 24}
\end{array}\right)-\operatorname{det}\left(\begin{array}{ll}
z_{, 11} & z_{, 12} \\
z_{, 13} & z_{, 23}
\end{array}\right) .
\end{aligned}
$$

$$
\begin{aligned}
& A_{1}^{4}=\operatorname{det}\left(\begin{array}{ll}
z_{, 22} & z_{, 23} \\
z_{, 24} & z_{, 34}
\end{array}\right)+\operatorname{det}\left(\begin{array}{ll}
z_{, 12} & z_{, 13} \\
z_{, 23} & z_{, 33}
\end{array}\right), \\
& A_{2}^{4}=-\operatorname{det}\left(\begin{array}{ll}
z_{, 12} & z_{, 14} \\
z_{, 23} & z_{, 34}
\end{array}\right)-\operatorname{det}\left(\begin{array}{ll}
z_{, 11} & z_{, 13} \\
z_{, 13} & z_{, 33}
\end{array}\right), \\
& A_{3}^{4}=\operatorname{det}\left(\begin{array}{ll}
z_{, 12} & z_{, 14} \\
z_{, 22} & z_{, 24}
\end{array}\right)+\operatorname{det}\left(\begin{array}{ll}
z_{, 11} & z_{, 12} \\
z_{, 13} & z_{, 23}
\end{array}\right) .
\end{aligned}
$$

A direct examination of the twelve operators in (2.25)-(2.28) gives the following result, which will be important in the sequel.

Lemma 2.6. Let $A_{i}^{j}$ be as in Lemma 2.4; then one has

$$
A_{i}^{j}+A_{j}^{i}=0
$$

We are now ready to prove the central result of this section.

Proof of Theorem 2.1. We begin as in the proof of Lemma2.3 and find as for (2.11),

$$
\begin{aligned}
f^{\prime}(s)= & \int_{\partial \Omega} \frac{\partial H(z)}{\partial z_{, i j}} \nu_{X, i} \nu_{X, j}\left|\nabla z_{s}\right| d \sigma \\
& -\int_{\Omega} X_{i} \frac{\partial H(z)}{\partial z_{, i j}} X_{j}\left(z_{s}\right) d g .
\end{aligned}
$$


To evaluate the solid integral in the right-hand side of (2.29) we first employ Lemma 2.4, and then integrate by parts obtaining

$$
\begin{aligned}
-\int_{\Omega} X_{i} \frac{\partial H(z)}{\partial z_{, i j}} X_{j}\left(z_{s}\right) d g & =-\frac{3}{2} \sum_{j=1}^{4} \sum_{\substack{i=1 \\
i \neq j}}^{4} \int_{\Omega} A_{i}^{j} X_{i}(T z) X_{j}\left(z_{s}\right) d g \\
= & -\frac{3}{2} \sum_{j=1}^{4} \sum_{\substack{i=1 \\
i \neq j}}^{4} \int_{\partial \Omega} A_{i}^{j}\left\langle X_{i}, \nu\right\rangle\left\langle X_{j}, \nu\right\rangle T z\left|\nabla z_{s}\right| d \sigma \\
& +\frac{3}{2} \sum_{j=1}^{4} \sum_{\substack{i=1 \\
i \neq j}}^{4} \int_{\Omega} A_{i}^{j} X_{i} X_{j}\left(z_{s}\right) T z d g \\
& +\frac{3}{2} \sum_{j=1}^{4} \sum_{\substack{i=1 \\
i \neq j}}^{4} \int_{\Omega} X_{i} A_{i}^{j} X_{j}\left(z_{s}\right) T z d g \\
= & (I)+(I I)+(I I I)
\end{aligned}
$$

where in the boundary integral we have used the relations (2.12), (2.14). We now claim that

$$
(I)=-\frac{3}{2} \sum_{j=1}^{4} \sum_{\substack{i=1 \\ i \neq j}}^{4} \int_{\partial \Omega} A_{i}^{j}\left\langle X_{i}, \nu\right\rangle\left\langle X_{j}, \nu\right\rangle T z\left|\nabla z_{s}\right| d \sigma=0 .
$$

This claim follows from the fact that, thanks to the skew-symmetry of the nonlinear coefficients $A_{i}^{j}$ guaranteed by Lemma 2.6. the factor

$$
\sum_{j=1}^{4} \sum_{\substack{i=1 \\ i \neq j}}^{4} A_{i}^{j}\left\langle X_{i}, \nu\right\rangle\left\langle X_{j}, \nu\right\rangle=\sum_{j=1}^{4} \sum_{\substack{i=1 \\ i \neq j}}^{4} A_{i}^{j} \nu_{X, i} \nu_{X, j}
$$

in the integrand in (2.31) vanishes indentically on $\partial \Omega$.

We then turn to evaluating $(I I)$. Using Lemma 2.4 again we see that the sum of the twelve terms in $(I I)$ reduces to the expression

$$
\begin{aligned}
(I I)= & \frac{3}{2} \sum_{j=1}^{4} \sum_{\substack{i=1 \\
i \neq j}}^{4} \int_{\Omega} A_{i}^{j} X_{i} X_{j}\left(z_{s}\right) T z d g \\
= & \frac{3}{2} \int_{\Omega}\left\{A_{2}^{1}\left[X_{2}, X_{1}\right]\left(z_{s}\right)+A_{3}^{1}\left[X_{3}, X_{1}\right]\left(z_{s}\right)+A_{4}^{1}\left[X_{4}, X_{1}\right]\left(z_{s}\right)\right. \\
& \left.+A_{3}^{2}\left[X_{3}, X_{2}\right]\left(z_{s}\right)+A_{4}^{2}\left[X_{4}, X_{2}\right]\left(z_{s}\right)+A_{4}^{3}\left[X_{4}, X_{3}\right]\left(z_{s}\right)\right\} T z d g \\
= & \frac{3}{2} \int_{\Omega}\left\{A_{3}^{1}\left[X_{3}, X_{1}\right]\left(z_{s}\right)+A_{4}^{2}\left[X_{4}, X_{2}\right]\left(z_{s}\right)\right\} T z d g \\
= & -\frac{3}{4} \int_{\Omega}\left(A_{3}^{1}+A_{4}^{2}\right) \frac{d}{d s}(T z)^{2} d g,
\end{aligned}
$$


where in the second-to-last equality we have used the trivial commutation relations $\left[X_{2}, X_{1}\right]=\left[X_{4}, X_{1}\right]=\left[X_{3}, X_{2}\right]=\left[X_{4}, X_{3}\right]=0$. From (2.25), (2.26) we find

$$
A_{3}^{1}+A_{4}^{2}=B+C
$$

where we have let

$$
B \stackrel{\text { def }}{=} \operatorname{det}\left(\begin{array}{ll}
z_{, 22} & z_{, 24} \\
z_{, 24} & z_{, 44}
\end{array}\right)+\operatorname{det}\left(\begin{array}{ll}
z_{, 11} & z, 13 \\
z, 13 & z, 33
\end{array}\right)
$$

$$
C \stackrel{\text { def }}{=} 2 \operatorname{det}\left(\begin{array}{ll}
z, 12 & z_{, 14} \\
z_{, 23} & z_{, 34}
\end{array}\right)
$$

We can thus rewrite (2.32) as follows:

$$
\begin{aligned}
(I I)= & -\frac{3}{4} \int_{\Omega}(B+C) \frac{d}{d s}(T z)^{2} d g \\
= & -\frac{d}{d s}\left(\frac{3}{4} \int_{\Omega}(B+C)(T z)^{2} d g\right) \\
& +\frac{3}{4} \int_{\Omega} \frac{d B}{d s}(T z)^{2} d g+\frac{3}{4} \int_{\Omega} \frac{d C}{d s}(T z)^{2} d g
\end{aligned}
$$

Substituting (2.31), (2.36) into (2.30), and using (2.29), we conclude that

$$
\begin{aligned}
\frac{d}{d s} \int_{\Omega} & \left\{H(z)+\frac{3}{4}(B+C)(T z)^{2}\right\} d g \\
= & \int_{\partial \Omega} \frac{\partial H(z)}{\partial z, i j} \nu_{X, i} \nu_{X, j}\left|\nabla z_{s}\right| d \sigma \\
& +\frac{3}{4} \int_{\Omega} \frac{d B}{d s}(T z)^{2} d g+\frac{3}{4} \int_{\Omega} \frac{d C}{d s}(T z)^{2} d g \\
& +\frac{3}{2} \sum_{j=1}^{4} \sum_{\substack{i=1 \\
i \neq j}}^{4} \int_{\Omega} X_{i} A_{i}^{j} X_{j}\left(z_{s}\right) T z d g \\
= & \int_{\partial \Omega} \frac{\partial H(z)}{\partial z_{, i j}} \nu_{X, i} \nu_{X, j}\left|\nabla z_{s}\right| d \sigma+\frac{3}{4} \text { Monster },
\end{aligned}
$$


where

Monster $=\int_{\Omega} \frac{d B}{d s}(T z)^{2} d g+\int_{\Omega} \frac{d C}{d s}(T z)^{2} d g+2 \sum_{j=1}^{4} \sum_{\substack{i=1 \\ i \neq j}}^{4} \int_{\Omega} X_{i} A_{i}^{j} X_{j}\left(z_{s}\right) T z d g$.

We now want to understand the Monster. We begin by computing the first integral in the right-hand side of (2.38). Using (2.34), and integration by parts, we find

$$
\begin{aligned}
& \int_{\Omega} \frac{d B}{d s}(T z)^{2} d g \\
= & \int_{\Omega}\left[\left(z_{s}\right)_{, 22} z_{, 44}+z_{, 22}\left(z_{s}\right)_{, 44}-2\left(z_{s}\right)_{, 24} z_{, 24}+\left(z_{s}\right)_{, 11} z_{, 33}\right. \\
& \left.+z_{, 11}\left(z_{s}\right)_{, 33}-2\left(z_{s}\right)_{, 13} z_{, 13}\right](T z)^{2} d g \\
= & \int_{\partial \Omega}\left[\left\langle\left(\begin{array}{cc}
z_{, 22} & z_{, 24} \\
z_{, 24} & z_{, 44}
\end{array}\right)\left(\begin{array}{c}
\nu_{X, 4} \\
-\nu_{X, 2}
\end{array}\right),\left(\begin{array}{c}
\nu_{X, 4} \\
-\nu_{X, 2}
\end{array}\right)\right\rangle\right. \\
& \left.+\left\langle\left(\begin{array}{cc}
z_{, 11} & z_{, 13} \\
z_{, 13} & z_{, 33}
\end{array}\right)\left(\begin{array}{c}
\nu_{X, 3} \\
-\nu_{X, 1}
\end{array}\right),\left(\begin{array}{c}
\nu_{X, 3} \\
-\nu_{X, 1}
\end{array}\right)\right\rangle\right]\left|\nabla z_{s}\right| d \sigma \\
- & \int_{\Omega}\left[X_{1}\left(z_{s}\right)\left(X_{1} z_{, 33}-X_{3} z_{, 13}\right)+X_{2}\left(z_{s}\right)\left(X_{2} z_{, 44}-X_{4} z_{, 24}\right)\right. \\
& \left.+X_{3}\left(z_{s}\right)\left(X_{3} z_{, 11}-X_{1} z_{, 13}\right)+X_{4}\left(z_{s}\right)\left(X_{4} z_{, 22}-X_{2} z_{, 24}\right)\right](T z)^{2} d g \\
- & 2 \int_{\Omega}\left[\left(z_{, 33} X_{1}(T z)-z_{, 13} X_{3}(T z)\right) X_{1}\left(z_{s}\right)+\left(z_{, 44} X_{2}(T z)-z_{, 24} X_{4}(T z)\right) X_{2}\left(z_{s}\right)\right. \\
& +\left(z_{, 11} X_{3}(T z)-z_{, 13} X_{1}(T z)\right) X_{3}\left(z_{s}\right) \\
& \left.+\left(z_{, 22} X_{4}(T z)-z_{, 24} X_{2}(T z)\right) X_{4}\left(z_{s}\right)\right] T z d g . \\
&
\end{aligned}
$$

Using (1.6) one recognizes that

$$
\begin{aligned}
X_{1} z_{, 33}-X_{3} z_{, 13} & =\frac{3}{2} X_{3}(T z), \\
X_{2} z_{, 44}-X_{4} z_{, 24} & =\frac{3}{2} X_{4}(T z), \\
X_{3} z_{, 11}-X_{1} z_{, 13} & =-\frac{3}{2} X_{1}(T z), \\
X_{4} z_{, 22}-X_{2} z_{, 24} & =-\frac{3}{2} X_{2}(T z) .
\end{aligned}
$$


Substituting (2.40) in the second integral on the right-hand side of (2.39) we find

$$
\begin{aligned}
& \int_{\Omega} \frac{d B}{d s}(T z)^{2} d g \\
= & \int_{\partial \Omega}\left[\left\langle\left(\begin{array}{cc}
z, 22 & z_{, 24} \\
z_{, 24} & z_{, 44}
\end{array}\right)\left(\begin{array}{c}
\nu_{X, 4} \\
-\nu_{X, 2}
\end{array}\right),\left(\begin{array}{c}
\nu_{X, 4} \\
-\nu_{X, 2}
\end{array}\right)\right\rangle\right. \\
& \left.+\left\langle\left(\begin{array}{ll}
z, 11 & z, 13 \\
z, 13 & z, 33
\end{array}\right)\left(\begin{array}{c}
\nu_{X, 3} \\
-\nu_{X, 1}
\end{array}\right),\left(\begin{array}{c}
\nu_{X, 3} \\
-\nu_{X, 1}
\end{array}\right)\right\rangle\right](T z)^{2}\left|\nabla z_{s}\right| d \sigma \\
- & \frac{3}{2} \int_{\Omega}\left[X_{3}(T z) X_{1}\left(z_{s}\right)+X_{4}(T z) X_{2}\left(z_{s}\right)\right. \\
& \left.-X_{1}(T z) X_{3}\left(z_{s}\right)-X_{2}(T z) X_{4}\left(z_{s}\right)\right](T z)^{2} d g \\
- & 2 \int_{\Omega}\left[\left(z, 33 X_{1}(T z)-z_{, 13} X_{3}(T z)\right) X_{1}\left(z_{s}\right)+\left(z_{, 44} X_{2}(T z)-z_{, 24} X_{4}(T z)\right) X_{2}\left(z_{s}\right)\right. \\
& +\left(z, 11 X_{3}(T z)-z_{, 13} X_{1}(T z)\right) X_{3}\left(z_{s}\right) \\
& \left.+\left(z_{, 22} X_{4}(T z)-z_{, 24} X_{2}(T z)\right) X_{4}\left(z_{s}\right)\right] T z d g .
\end{aligned}
$$

We next compute the second integral on the right-hand side of (2.41). An integration by parts gives

$$
\begin{aligned}
& \int_{\Omega}\left[X_{3}(T z) X_{1}\left(z_{s}\right)+X_{4}(T z) X_{2}\left(z_{s}\right)\right. \\
& \left.-X_{1}(T z) X_{3}\left(z_{s}\right)-X_{2}(T z) X_{4}\left(z_{s}\right)\right](T z)^{2} d g \\
& =\int_{\partial \Omega}\left[\left\langle X_{3}, \nu\right\rangle\left\langle X_{1}, \nu\right\rangle+\left\langle X_{4}, \nu\right\rangle\left\langle X_{2}, \nu\right\rangle\right. \\
& \left.+-\left\langle X_{1}, \nu\right\rangle\left\langle X_{3}, \nu\right\rangle-\left\langle X_{2}, \nu\right\rangle\left\langle X_{4}, \nu\right\rangle\right](T z)^{3}\left|\nabla z_{s}\right| d \sigma \\
& -\int_{\Omega}\left[X_{3} X_{1}\left(z_{s}\right)+X_{4} X_{2}\left(z_{s}\right)-X_{1} X_{3}\left(z_{s}\right)-X_{2} X_{4}\left(z_{s}\right)\right](T z)^{3} d g \\
& -2 \int_{\Omega}\left[X_{3}(T z) X_{1}\left(z_{s}\right)+X_{4}(T z) X_{2}\left(z_{s}\right)\right. \\
& \left.-X_{1}(T z) X_{3}\left(z_{s}\right)-X_{2}(T z) X_{4}\left(z_{s}\right)\right](T z)^{2} d g .
\end{aligned}
$$

In this computation some miracles have occurred. First of all, thanks to the special symmetries of the integrand, the boundary integral on the right-hand side of (2.42) disappears. Furthermore, thanks to the commutation relations (1.6), the second term is a pure derivative with respect to $s$. Finally, the left-hand side (which is what we want to compute) appears with the opposite sign with respect to the 
third integral on the right-hand side of (2.42). Solving for such term, we thus obtain

$$
\begin{aligned}
& \int_{\Omega}\left[X_{3}(T z) X_{1}\left(z_{s}\right)+X_{4}(T z) X_{2}\left(z_{s}\right)\right. \\
& \left.-X_{1}(T z) X_{3}\left(z_{s}\right)-X_{2}(T z) X_{4}\left(z_{s}\right)\right](T z)^{2} d g \\
& =\frac{1}{6} \frac{d}{d s} \int_{\Omega}(T z)^{4} d g .
\end{aligned}
$$

Armed with (2.43) we return to (2.41) to conclude

$$
\begin{aligned}
& \int_{\Omega} \frac{d B}{d s}(T z)^{2} d g=-\frac{3}{12} \frac{d}{d s} \int_{\Omega}(T z)^{4} d g \\
+ & \int_{\partial \Omega}\left[\left\langle\left(\begin{array}{cc}
z, 22 & z_{, 24} \\
z, 24 & z_{, 44}
\end{array}\right)\left(\begin{array}{c}
\nu_{X, 4} \\
-\nu_{X, 2}
\end{array}\right),\left(\begin{array}{c}
\nu_{X, 4} \\
-\nu_{X, 2}
\end{array}\right)\right\rangle\right. \\
& \left.+\left\langle\left(\begin{array}{cc}
z, 11 & z_{, 13} \\
z, 13 & z_{, 33}
\end{array}\right)\left(\begin{array}{c}
\nu_{X, 3} \\
-\nu_{X, 1}
\end{array}\right),\left(\begin{array}{c}
\nu_{X, 3} \\
-\nu_{X, 1}
\end{array}\right)\right\rangle\right](T z)^{2}\left|\nabla z_{s}\right| d \sigma \\
- & 2 \int_{\Omega}\left[\left(z_{, 33} X_{1}(T z)-z_{, 13} X_{3}(T z)\right) X_{1}\left(z_{s}\right)+\left(z_{, 44} X_{2}(T z)-z_{, 24} X_{4}(T z)\right) X_{2}\left(z_{s}\right)\right. \\
& +\left(z, 11 X_{3}(T z)-z_{, 13} X_{1}(T z)\right) X_{3}\left(z_{s}\right) \\
& \left.+\left(z_{, 22} X_{4}(T z)-z_{, 24} X_{2}(T z)\right) X_{4}\left(z_{s}\right)\right] T z d g .
\end{aligned}
$$

This gives the first piece of the Monster (2.38). We next compute the second piece. Using (2.35) and integration by parts we find

$$
\begin{aligned}
& \int_{\Omega} \frac{d C}{d s}(T z)^{2} d g \\
= & 2 \int_{\Omega}\left[\left(z_{s}\right)_{, 12} z_{, 34}+z_{, 12}\left(z_{s}\right)_{, 34}-\left(z_{s}\right)_{, 14} z_{, 23}-z_{, 14}\left(z_{s}\right)_{, 23}\right](T z)^{2} d g \\
= & \int_{\partial \Omega}\left[2 z_{, 34} \nu_{X, 1} \nu_{X, 2}+2 z_{, 12} \nu_{X, 3} \nu_{X, 4}-2 z_{, 23} \nu_{X, 1} \nu_{X, 4}-2 z_{, 14} \nu_{X, 2} \nu_{X, 3}\right](T z)^{2}\left|\nabla z_{s}\right| d \sigma \\
- & \int_{\Omega}\left[X_{1}\left(z_{s}\right)\left(X_{2} z_{, 34}-X_{4} z_{, 23}\right)+X_{2}\left(z_{s}\right)\left(X_{1} z_{, 34}-X_{3} z_{, 14}\right)\right. \\
& \left.+X_{3}\left(z_{s}\right)\left(X_{4} z_{, 12}-X_{2} z_{, 14}\right)+X_{4}\left(z_{s}\right)\left(X_{3} z_{, 12}-X_{1} z_{, 23}\right)\right](T z)^{2} d g \\
- & 2 \int_{\Omega}\left[\left(z_{, 34} X_{2}(T z)-z_{, 23} X_{4}(T z)\right) X_{1}\left(z_{s}\right)+\left(z_{, 34} X_{1}(T z)-z_{, 14} X_{3}(T z)\right) X_{2}\left(z_{s}\right)\right. \\
& \left.\left(z, 12 X_{4}(T z)-z_{, 14} X_{2}(T z)\right) X_{3}\left(z_{s}\right)+\left(z_{, 12} X_{3}(T z)-z_{, 23} X_{1}(T z)\right) X_{4}\left(z_{s}\right)\right] T z d g .
\end{aligned}
$$

From (1.5) we recognize that

$$
\begin{aligned}
& X_{2} z_{, 34}-X_{4} z_{, 23}=X_{3}(T z), \\
& X_{1} z_{, 34}-X_{3} z_{, 14}=X_{4}(T z), \\
& X_{4} z_{, 12}-X_{2} z_{, 14}=-X_{1}(T z), \\
& X_{3} z_{, 12}-X_{1} z_{, 23}=-X_{2}(T z) .
\end{aligned}
$$


Substituting (2.46) in the second integral on the right-hand side of (2.45) we obtain

$$
\begin{aligned}
& \int_{\Omega} \frac{d C}{d s}(T z)^{2} d g \\
= & \int_{\partial \Omega}\left[2 z_{, 34} \nu_{X, 1} \nu_{X, 2}+2 z, 12 \nu_{X, 3} \nu_{X, 4}-2 z_{, 23} \nu_{X, 1} \nu_{X, 4}\right. \\
& \left.-2 z z_{, 14} \nu_{X, 2} \nu_{X, 3}\right](T z)^{2}\left|\nabla z_{s}\right| d \sigma \\
- & \int_{\Omega}\left[X_{1}\left(z_{s}\right) X_{3}(T z)+X_{2}\left(z_{s}\right) X_{4}(T z)-X_{3}\left(z_{s}\right) X_{1}(T z)\right. \\
& \left.-X_{4}\left(z_{s}\right) X_{2}(T z)\right](T z)^{2} d g \\
- & 2 \int_{\Omega}\left[\left(z_{, 34} X_{2}(T z)-z_{, 23} X_{4}(T z)\right) X_{1}\left(z_{s}\right)+\left(z_{, 34} X_{1}(T z)-z_{, 14} X_{3}(T z)\right) X_{2}\left(z_{s}\right)\right. \\
& +\left(z, 12 X_{4}(T z)-z_{, 14} X_{2}(T z)\right) X_{3}\left(z_{s}\right) \\
& \left.+\left(z_{, 12} X_{3}(T z)-z_{, 23} X_{1}(T z)\right) X_{4}\left(z_{s}\right)\right] T z d g .
\end{aligned}
$$

At this point we need to compute the second integral on the right-hand side of (2.47). But this has already been done in (2.42). We can thus use (2.43) and conclude from (2.47) that

$$
\begin{aligned}
& \int_{\Omega} \frac{d C}{d s}(T z)^{2} d g=-\frac{1}{6} \frac{d}{d s} \int_{\Omega}(T z)^{4} d g \\
+ & \int_{\partial \Omega}\left[2 z_{, 34} \nu_{X, 1} \nu_{X, 2}+2 z_{, 12} \nu_{X, 3} \nu_{X, 4}-2 z_{, 23} \nu_{X, 1} \nu_{X, 4}\right. \\
& \left.-2 z_{, 14} \nu_{X, 2} \nu_{X, 3}\right](T z)^{2}\left|\nabla z_{s}\right| d \sigma \\
- & 2 \int_{\Omega}\left[\left(z_{, 34} X_{2}(T z)-z_{, 23} X_{4}(T z)\right) X_{1}\left(z_{s}\right)+\left(z_{, 34} X_{1}(T z)-z_{, 14} X_{3}(T z)\right) X_{2}\left(z_{s}\right)\right. \\
& +\left(z_{, 12} X_{4}(T z)-z_{, 14} X_{2}(T z)\right) X_{3}\left(z_{s}\right) \\
& \left.+\left(z_{, 12} X_{3}(T z)-z_{, 23} X_{1}(T z)\right) X_{4}\left(z_{s}\right)\right] T z d g .
\end{aligned}
$$


Formula (2.48) gives the second piece of the Monster. Inserting (2.44) and (2.48) in (2.38) we conclude that

$$
\begin{aligned}
& \text { Monster }=-\frac{d}{d s} \frac{5}{12} \int_{\Omega}(T z)^{4} d g+2 \sum_{j=1}^{4} \sum_{\substack{i=1 \\
i \neq j}}^{4} \int_{\Omega} X_{i} A_{i}^{j} X_{j}\left(z_{s}\right) T z d g \\
+ & \int_{\partial \Omega}\left[\left\langle\left(\begin{array}{cc}
z, 22 & z_{, 24} \\
z, 24 & z_{, 44}
\end{array}\right)\left(\begin{array}{c}
\nu_{X, 4} \\
-\nu_{X, 2}
\end{array}\right),\left(\begin{array}{c}
\nu_{X, 4} \\
-\nu_{X, 2}
\end{array}\right)\right\rangle\right. \\
& \left.+\left\langle\left(\begin{array}{cc}
z, 11 & z_{, 13} \\
z, 13 & z_{, 33}
\end{array}\right)\left(\begin{array}{c}
\nu_{X, 3} \\
-\nu_{X, 1}
\end{array}\right),\left(\begin{array}{c}
\nu_{X, 3} \\
-\nu_{X, 1}
\end{array}\right)\right\rangle\right](T z)^{2}\left|\nabla z_{s}\right| d \sigma \\
+ & \int_{\partial \Omega}\left[2 z_{, 34} \nu_{X, 1} \nu_{X, 2}+2 z_{, 12} \nu_{X, 3} \nu_{X, 4}-2 z_{, 23} \nu_{X, 1} \nu_{X, 4}-2 z_{, 14} \nu_{X, 2} \nu_{X, 3}\right](T z)^{2}\left|\nabla z_{s}\right| d \sigma \\
- & 2 \int_{\Omega}\left[\left(z, 33 X_{1}(T z)-z_{, 13} X_{3}(T z)\right) X_{1}\left(z_{s}\right)+\left(z_{, 44} X_{2}(T z)-z_{, 24} X_{4}(T z)\right) X_{2}\left(z_{s}\right)\right. \\
& \left.+\left(z_{, 11} X_{3}(T z)-z, 13 X_{1}(T z)\right) X_{3}\left(z_{s}\right)+\left(z_{, 22} X_{4}(T z)-z_{, 24} X_{2}(T z)\right) X_{4}\left(z_{s}\right)\right] T z d g \\
- & 2 \int_{\Omega}\left[\left(z_{, 34} X_{2}(T z)-z_{, 23} X_{4}(T z)\right) X_{1}\left(z_{s}\right)+\left(z_{, 34} X_{1}(T z)-z_{, 14} X_{3}(T z)\right) X_{2}\left(z_{s}\right)\right. \\
& \left.+\left(z_{, 12} X_{4}(T z)-z_{, 14} X_{2}(T z)\right) X_{3}\left(z_{s}\right)+\left(z_{, 12} X_{3}(T z)-z_{, 23} X_{1}(T z)\right) X_{4}\left(z_{s}\right)\right] T z d g .
\end{aligned}
$$

At this point two remarkable facts occurr. First, if we consider the vector field on $\partial \Omega, \nu \frac{1}{X}$, introduced in (2.4), a computation shows that sum of the integrands in the two boundary integrals in (2.49) equals $\left\langle\operatorname{Hess}_{X}(z) \nu \frac{1}{X}, \nu_{X}^{\frac{1}{X}}\right\rangle$, i.e., we have

$$
\begin{aligned}
& \int_{\partial \Omega}\left\{\left\langle\left(\begin{array}{cc}
z_{, 22} & z_{, 24} \\
z, 24 & z_{, 44}
\end{array}\right)\left(\begin{array}{c}
\nu_{X, 4} \\
-\nu_{X, 2}
\end{array}\right),\left(\begin{array}{c}
\nu_{X, 4} \\
-\nu_{X, 2}
\end{array}\right)\right\rangle\right. \\
+ & \left\langle\left(\begin{array}{cc}
z, 11 & z, 13 \\
z, 13 & z_{, 33}
\end{array}\right)\left(\begin{array}{c}
\nu_{X, 3} \\
-\nu_{X, 1}
\end{array}\right),\left(\begin{array}{c}
\nu_{X, 3} \\
-\nu_{X, 1}
\end{array}\right)\right\rangle \\
+ & \left.2 z_{, 34} \nu_{X, 1} \nu_{X, 2}+2 z_{, 12} \nu_{X, 3} \nu_{X, 4}-2 z_{, 23} \nu_{X, 1} \nu_{X, 4}-2 z_{, 14} \nu_{X, 2} \nu_{X, 3}\right\}(T z)^{2}\left|\nabla z_{s}\right| d \sigma \\
= & \int_{\partial \Omega}\left\langle\operatorname{Hess}_{X}(z) \nu_{X}^{\perp}, \nu_{X}^{\perp}\right\rangle(T z)^{2}\left|\nabla z_{s}\right| d \sigma .
\end{aligned}
$$

The second (big) miracle is contained in the following lemma. 
Lemma 2.7. The following identity holds:

$$
\begin{aligned}
& \sum_{j=1}^{4} \sum_{\substack{i=1 \\
i \neq j}}^{4} X_{i} A_{i}^{j} X_{j}\left(z_{s}\right) \\
& =\left(z_{, 33} X_{1}(T z)-z_{, 13} X_{3}(T z)\right) X_{1}\left(z_{s}\right)+\left(z_{, 44} X_{2}(T z)-z_{, 24} X_{4}(T z)\right) X_{2}\left(z_{s}\right) \\
& -\left(z, 11 X_{3}(T z)-z_{, 13} X_{1}(T z)\right) X_{3}\left(z_{s}\right)+\left(z_{, 22} X_{4}(T z)-z_{, 24} X_{2}(T z)\right) X_{4}\left(z_{s}\right) \\
& +\left(z, 34 X_{2}(T z)-z_{, 23} X_{4}(T z)\right) X_{1}\left(z_{s}\right)+\left(z_{, 34} X_{1}(T z)-z_{, 14} X_{3}(T z)\right) X_{2}\left(z_{s}\right) \\
& -\left(z_{, 12} X_{4}(T z)-z_{, 14} X_{2}(T z)\right) X_{3}\left(z_{s}\right)+\left(z_{, 12} X_{3}(T z)-z_{, 23} X_{1}(T z)\right) X_{4}\left(z_{s}\right) .
\end{aligned}
$$

Proof. We have

$$
\begin{aligned}
\sum_{j=1}^{4} \sum_{\substack{i \neq j \\
i \neq j}}^{4} X_{i} A_{i}^{j} X_{j}\left(z_{s}\right) & =\left(X_{2} A_{2}^{1}+X_{3} A_{3}^{1}+X_{4} A_{4}^{1}\right) X_{1}\left(z_{s}\right) \\
& +\left(X_{1} A_{1}^{2}+X_{3} A_{3}^{2}+X_{4} A_{4}^{2}\right) X_{2}\left(z_{s}\right) \\
& +\left(X_{1} A_{1}^{3}+X_{2} A_{2}^{3}+X_{4} A_{4}^{3}\right) X_{3}\left(z_{s}\right) \\
& +\left(X_{1} A_{1}^{4}+X_{2} A_{2}^{4}+X_{3} A_{3}^{4}\right) X_{4}\left(z_{s}\right) .
\end{aligned}
$$

The computation of the four terms on the right-hand side of (2.52) is very long, therefore we omit the tedious details and confine ourselves to provide the reader with the final output:

$$
\begin{aligned}
& X_{2} A_{2}^{1}+X_{3} A_{3}^{1}+X_{4} A_{4}^{1}=z_{, 33} X_{1}(T z)+z_{, 34} X_{2}(T z)-z_{, 13} X_{3}(T z)-z_{, 23} X_{4}(T z), \\
& X_{1} A_{1}^{2}+X_{3} A_{3}^{2}+X_{4} A_{4}^{2}=z_{, 34} X_{1}(T z)+z_{, 44} X_{2}(T z)-z_{, 14} X_{3}(T z)-z_{, 24} X_{4}(T z), \\
& X_{1} A_{1}^{3}+X_{2} A_{2}^{3}+X_{4} A_{4}^{3}=-z_{, 13} X_{1}(T z)-z_{, 14} X_{2}(T z)+z_{, 11} X_{3}(T z)+z_{, 12} X_{4}(T z), \\
& X_{1} A_{1}^{4}+X_{2} A_{2}^{4}+X_{3} A_{3}^{4}=-z_{, 23} X_{1}(T z)-z_{, 24} X_{2}(T z)+z_{, 12} X_{3}(T z)+z_{, 22} X_{4}(T z) .
\end{aligned}
$$

To finish the proof of the lemma all we need to do at this point is to substitute the expressions of the four terms in (2.53) in the corresponding equations (2.52), and then recognize that the resulting expression equals the right-hand side of (2.51).

Armed with Lemma 2.7 we can now complete the proof of Theorem 2.1. It suffices to insert (2.50) and (2.51) in (2.49) to find

$$
\text { Monster }=-\frac{d}{d s} \frac{5}{12} \int_{\Omega}(T z)^{4} d g+\int_{\partial \Omega}\left\langle\operatorname{Hess}_{X}(z) \nu_{X}^{\perp}, \nu_{X}^{\perp}\right\rangle(T z)^{2}\left|\nabla z_{s}\right| d \sigma .
$$

Finally, substitution of (2.54) in (2.37) allows us to reach the sought-for conclusion.

We close this section by formulating a conjecture for a monotonicity result for general $n$. We plan to come back to this conjecture in a forthcoming study. 
Conjecture. Let $\Omega \subset \mathbb{H}^{n}$ be a $C^{1}$ domain, and consider two weakly $H$-convex functions $u, v \in \Gamma^{3}(\bar{\Omega})$ such that $u \geq v$ in $\Omega$ and $u=v$ on $\partial \Omega$. With $z$ as in (2.9) one has for suitable numbers $a_{n, k}>0$

$$
\frac{d}{d s} \int_{\Omega}\left\{\operatorname{det} \operatorname{Hess}_{X}(z)+\sum_{k=1}^{n} a_{n, k}\left(\sum(\text { Minors })_{2(n-k) \times 2(n-k)}\right)(T z)^{2 k}\right\} d g \geq 0 .
$$

Here, the sum is extended to suitably selected minors of order $2(n-k) \times 2(n-k)$ of the matrix $\operatorname{Hess}_{X}(u)$.

\section{Positivity of the Monge-Ampère measure}

For a function $u \in \Gamma^{2}\left(\mathbb{H}^{2}\right)$ we consider the modified sub-elliptic Monge-Ampère operator $\mathcal{S}_{m a}(u)$ defined in (1.7). Given a weakly $H$-convex $u \in \Gamma^{2}\left(\mathbb{H}^{2}\right)$, we now introduce a measure on $\mathbb{H}^{2}$ as follows:

$$
\nu_{m a}(E) \stackrel{\text { def }}{=} \int_{E} \mathcal{S}_{m a}(u) d g, \quad E \subset \mathbb{H}^{n} \quad \text { is a Borel set } .
$$

In this section we are interested in a basic property of such a measure which will play an important role in Section 7, namely, its positivity. Clearly, when $n=1$, then such positivity is trivially guaranteed by Theorem 2.2. However, when $n=2$ such a basic property is a priori very much in doubt. Our next result shows that this property is in fact true for arbitrary (smooth) weakly $H$-convex functions. The proof of the following linear algebra lemma was kindly suggested to us by Duy-Minh Nhieu, and we thank him for his help.

Lemma 3.1. Let $U=\left(u_{i j}\right)$ be a $4 \times 4$ symmetric, positive semi-definite matrix. Then the quantity

$$
\left(u_{11} u_{33}-u_{13}^{2}\right)+\left(u_{22} u_{44}-u_{24}^{2}\right)+2\left(u_{12} u_{34}-u_{14} u_{23}\right) \geq 0 .
$$

Proof. We begin by recalling that, according to the Cholesky factorization (see for instance $\mathrm{Hou}$ ), every symmetric, positive semi-definite matrix $U=\left(u_{i j}\right)$ can be written as follows: $U=L L^{t}$, where $L$ is a lower-triangular matrix and $L^{t}$ denotes its transpose. Denoting by $L_{i}$ the $i$-th row of $L$, we thus have $u_{i j}=\left\langle L_{i}, L_{j}\right\rangle$. The inequality (3.2) is thus equivalent to

$$
\begin{aligned}
& \left\{\left|L_{1}\right|^{2}\left|L_{3}\right|^{2}-\left\langle L_{1}, L_{3}\right\rangle^{2}\right\}+\left\{\left|L_{2}\right|^{2}\left|L_{4}\right|^{2}-\left\langle L_{2}, L_{4}\right\rangle^{2}\right\} \\
& +2\left\{\left\langle L_{1}, L_{2}\right\rangle\left\langle L_{3}, L_{4}\right\rangle-\left\langle L_{1}, L_{4}\right\rangle\left\langle L_{2}, L_{3}\right\rangle\right\} \geq 0 .
\end{aligned}
$$

We can presently write

$$
\begin{aligned}
& L_{1}=\left(l_{11}, 0,0,0\right), L_{2}=\left(l_{21}, l_{22}, 0,0\right) \\
& L_{3}=\left(l_{31}, l_{32}, l_{33}, 0\right), L_{4}=\left(l_{41}, l_{42}, l_{43}, l_{44}\right) .
\end{aligned}
$$


With this notation we find

$$
\left\{\left|L_{1}\right|^{2}\left|L_{3}\right|^{2}-\left\langle L_{1}, L_{3}\right\rangle^{2}\right\}=l_{11}^{2}\left(l_{31}^{2}+l_{32}^{2}+l_{33}^{2}\right)-l_{11}^{2} l_{31}^{2}=l_{11}^{2} l_{32}^{2}+l_{11}^{2} l_{33}^{2},
$$

$$
\begin{aligned}
& \left\{\left|L_{2}\right|^{2}\left|L_{4}\right|^{2}-\left\langle L_{2}, L_{4}\right\rangle^{2}\right\}=\left(l_{21}^{2}+l_{22}^{2}\right)\left(l_{41}^{2}+l_{42}^{2}+l_{43}^{2}+l_{44}^{2}\right)-\left(l_{21} l_{41}+l_{22} l_{42}\right)^{2} \\
& =\left(l_{21} l_{42}-l_{22} l_{41}\right)^{2}+l_{21}^{2} l_{44}^{2}+l_{21}^{2} l_{43}^{2}+l_{22}^{2} l_{43}^{2}+l_{22}^{2} l_{44}^{2} .
\end{aligned}
$$

$$
\begin{aligned}
2 & \left\{\left\langle L_{1}, L_{2}\right\rangle\left\langle L_{3}, L_{4}\right\rangle-\left\langle L_{1}, L_{4}\right\rangle\left\langle L_{2}, L_{3}\right\rangle\right\} \\
= & 2\left[l_{11} l_{21}\left(l_{31} l_{41}+l_{32} l_{42}+l_{33} l_{43}\right)-l_{11} l_{41}\left(l_{21} l_{31}+l_{22} l_{32}\right)\right] \\
= & 2 l_{11} l_{21} l_{33} l_{43}+2 l_{11} l_{21} l_{32} l_{42}-2 l_{11} l_{41} l_{22} l_{32} .
\end{aligned}
$$

Using the inequality $2|a b| \leq a^{2}+b^{2}$, we now infer that

$$
2 l_{11} l_{21} l_{33} l_{43} \leq l_{11}^{2} l_{33}^{2}+l_{21}^{2} l_{43}^{2} .
$$

Using this inequality, and comparing (3.4)-(3.6), we see that for (3.3) to hold, it suffices to have

$$
\begin{aligned}
& \left|2 l_{11} l_{21} l_{32} l_{42}-2 l_{11} l_{41} l_{22} l_{32}\right| \\
& \leq l_{11}^{2} l_{32}^{2}+\left(l_{21} l_{42}-l_{22} l_{41}\right)^{2}+l_{21}^{2} l_{44}^{2}+l_{22}^{2} l_{43}^{2}+l_{22}^{2} l_{44}^{2} .
\end{aligned}
$$

Since $2|a b| \leq a^{2}+b^{2}$ implies

$$
\left|2 l_{11} l_{21} l_{32} l_{42}-2 l_{11} l_{41} l_{22} l_{32}\right| \leq l_{11}^{2} l_{32}^{2}+\left(l_{21} l_{42}-l_{22} l_{41}\right)^{2},
$$

the validity of (3.7) now holds trivially. This completes the proof.

Now consider a function $u \in \Gamma^{2}\left(\mathbb{H}^{2}\right)$. According to Theorem 2.2 the matrix $\operatorname{Hess}_{X}(u)$ is positive semi-definite. Applying Lemma 3.1 to such a matrix, we obtain the following result.

Theorem 3.2. Let $u \in \Gamma^{2}\left(\mathbb{H}^{2}\right)$ be a weakly $H$-convex function. Then

$$
\operatorname{det}\left(\begin{array}{ll}
u_{, 22} & u_{, 24} \\
u_{, 24} & u_{, 44}
\end{array}\right)+2 \operatorname{det}\left(\begin{array}{ll}
u_{, 12} & u_{, 14} \\
u_{, 23} & u_{, 34}
\end{array}\right)+\operatorname{det}\left(\begin{array}{ll}
u_{, 11} & u_{, 13} \\
u_{, 13} & u_{, 33}
\end{array}\right) \geq 0 .
$$

In particular, from (3.8), from Theorem 2.2, and definition (1.7), we obtain

$$
\mathcal{S}_{m a}(u) \geq \frac{5}{16}(T u)^{4} \geq 0 .
$$

\section{Monotonicity for the Engel Group of Step three}

In this section we generalize Theorem 1.1 to an interesting four-dimensional Carnot group of step $r=3$, the so-called cyclic or Engel group. This group is important in many respects since it represents the next level of difficulty with respect to the Heisenberg group and provides an ideal framework for testing whether results which are true in step 2 generalize to step 3 or higher. The reader unfamiliar with the cyclic group can consult [CGr, or also Mon]. As we will see, because of the nonvanishing higher commutators (see (4.1)), Theorem 1.1 does not hold in the same form for such a group. Instead, we will find a substitute monotonicity result and a suitable expression for the Monge-Ampère measure. We mention here that in the forthcoming paper DGNT we will take up the ideas in the proof of Theorem 4.1 and establish a useful generalization of this result to arbitrary Carnot groups. 
The Engel group $\mathfrak{E}=K_{3}$ (see ex. 1.1.3 in [CGr] ) is the Lie group whose underlying manifold can be identified with $\mathbb{R}^{4}$, and whose Lie algebra is given by the grading,

$$
\mathfrak{e}=V_{1} \oplus V_{2} \oplus V_{3},
$$

where $V_{1}=\operatorname{span}\left\{e_{1}, e_{2}\right\}, V_{2}=\operatorname{span}\left\{e_{3}\right\}$, and $V_{3}=\operatorname{span}\left\{e_{4}\right\}$, so that $m_{1}=2$ and $m_{2}=m_{3}=1$. We will denote with $(x, y), t$ and $s$ respectively, the variables in $V_{1}$, $V_{2}$ and $V_{3}$, so that $X \in \mathfrak{e}$ can be written as $X=x e_{1}+y e_{2}+t e_{3}+s e_{4}$. If $g=\exp (X)$, we will identify $g=(x, y, t, s)$. For the corresponding left-invariant vector fields on $\mathfrak{E}$ given by $X_{i}(g)=\left(L_{g}\right)_{*}\left(e_{i}\right), i=1, \ldots, 4$, we assign the commutators

$$
\left[X_{1}, X_{2}\right]=X_{3}, \quad\left[X_{1}, X_{3}\right]=\left[X_{1},\left[X_{1}, X_{2}\right]\right]=X_{4},
$$

all other commutators being assumed trivial. We observe right away that the homogeneous dimension of $\mathfrak{E}$ is

$$
Q=m_{1}+2 m_{2}+3 m_{3}=7 .
$$

The group law in $\mathfrak{E}$ is given by the Baker-Campbell-Hausdorff formula [V]. In exponential coordinates, if $g=\exp (X), g^{\prime}=\exp \left(X^{\prime}\right)$, where $X=\sum_{i=1}^{4} x_{i} X_{i}$, $X^{\prime}=\sum_{i=1}^{4} y_{i} X_{i}$, we have

$$
g \circ g^{\prime}=X+X^{\prime}+\frac{1}{2}\left[X, X^{\prime}\right]+\frac{1}{12}\left\{\left[X,\left[X, X^{\prime}\right]\right]-\left[X^{\prime},\left[X, X^{\prime}\right]\right]\right\} .
$$

A computation based on (4.1) gives (see also ex. 1.2.5 in $\mathrm{CGr}$ )

$$
g \circ g^{\prime}=\left(x+x^{\prime}, y+y^{\prime}, t+t^{\prime}+P_{3}, s+s^{\prime}+P_{4}\right) \text {, }
$$

where

$$
\begin{gathered}
P_{3}=\frac{1}{2}\left(x y^{\prime}-y x^{\prime}\right) \\
P_{4}=\frac{1}{2}\left(x t^{\prime}-t x^{\prime}\right)+\frac{1}{12}\left(x^{2} y^{\prime}-x x^{\prime}\left(y+y^{\prime}\right)+y x^{\prime 2}\right) .
\end{gathered}
$$

Using the Baker-Campbell-Hausdorff formula we find the following expressions for the vector fields $X_{1}, \ldots, X_{4}$ :

$$
\begin{aligned}
X_{1} & =\frac{\partial}{\partial x}-\frac{y}{2} \frac{\partial}{\partial t}-\left(\frac{t}{2}+\frac{x y}{12}\right) \frac{\partial}{\partial s} \\
X_{2} & =\frac{\partial}{\partial y}+\frac{x}{2} \frac{\partial}{\partial t}+\frac{x^{2}}{12} \frac{\partial}{\partial s} \\
X_{3} & =\frac{\partial}{\partial t}+\frac{x}{2} \frac{\partial}{\partial s} \\
X_{4} & =\frac{\partial}{\partial s} .
\end{aligned}
$$

We define $\operatorname{Hess}_{X}(u)=\left(u_{, i j}\right)_{i, j=1,2}$, and we let $H(u)=\operatorname{det}\left(\operatorname{Hess}_{X}(u)\right)$; see (2.5).

Theorem 4.1. Let $\Omega \subset \mathfrak{E}$ be a $C^{1}$ bounded open set, and consider two weakly $H$-convex functions $u, v \in \Gamma^{3}(\bar{\Omega})$ such that $u \geq v$ in $\Omega$ and $u=v$ on $\partial \Omega$. For $0 \leq \tau \leq 1$ we set

$$
z=z(g, \tau) \stackrel{\text { def }}{=}(1-\tau) u(g)+\tau v(g), \quad g \in \Omega .
$$


We have

$$
\frac{d}{d \tau} \int_{\Omega}\left\{H(z)+\frac{3}{4}\left(X_{3} z\right)^{2}+\frac{1}{2}\left(X_{2} z\right)\left(X_{4} z\right)\right\} d g \geq 0
$$

In particular,

$$
\begin{aligned}
& \int_{\Omega}\left\{H(u)+\frac{3}{4}\left(X_{3} u\right)^{2}+\frac{1}{2}\left(X_{2} u\right)\left(X_{4} u\right)\right\} d g \\
& \leq \int_{\Omega}\left\{H(v)+\frac{3}{4}\left(X_{3} v\right)^{2}+\frac{1}{2}\left(X_{2} v\right)\left(X_{4} v\right)\right\} d g .
\end{aligned}
$$

Proof. We observe preliminarily that by the assumption $v, v \in \Gamma^{3}(\bar{\Omega})$ and the commutation relations (4.1), we have $X_{2} u, X_{3} u, X_{4} u, X_{4} v \in C(\bar{\Omega})$, and this implies in particular that $u, v \in C^{1}(\bar{\Omega})$. We can thus apply the divergence theorem to the function $g \rightarrow z(g, s)$. This being said, the proof now proceeds similarly to that of Theorem 2.1 up to formula (2.11), which we record again

$$
f^{\prime}(\tau)=\int_{\partial \Omega} \frac{\partial H(z)}{\partial z_{, i j}} \nu_{X, i} \nu_{X, j}\left|\nabla z_{\tau}\right| d \sigma-\int_{\Omega} X_{i} \frac{\partial H(z)}{\partial z_{, i j}} X_{j}\left(z_{\tau}\right) d g
$$

To evaluate the solid integral on the right-hand side of (4.3) we proceed as before, except that, since the first layer $V_{1}$ of the Engel Lie algebra is only twodimensional, similarly to the case of $\mathbb{H}^{1}$, the expression of $H(z)=z_{, 11} z_{, 22}-z_{, 12} z_{, 12}$ is much simpler. We thus find

$$
\begin{aligned}
X_{i} \frac{\partial H(z)}{\partial z_{, i j}} X_{j}\left(z_{s}\right)=\{ & \left.X_{1} X_{2} X_{2} z-X_{2}(z, 12)\right\} X_{1}\left(z_{\tau}\right) \\
& +\left\{-X_{1}(z, 12)+X_{2} X_{1} X_{1} z\right\} X_{2}\left(z_{\tau}\right) .
\end{aligned}
$$

At this point we use (4.1) to find

$$
\begin{gathered}
X_{1} X_{2} X_{2} z-X_{2}(z, 12)=\frac{3}{2} X_{2}\left(X_{3} z\right) \\
-X_{1}\left(z_{, 12}\right)+X_{2} X_{1} X_{1} z=-\frac{3}{2} X_{1}\left(X_{3} z\right)+X_{4} z .
\end{gathered}
$$

Substituting (4.4), (4.5) in the above formula, we conclude that

$$
X_{i} \frac{\partial H(z)}{\partial z_{, i j}} X_{j}\left(z_{\tau}\right)=\frac{3}{2} X_{2}\left(X_{3} z\right) X_{1}\left(z_{\tau}\right)-\frac{3}{2} X_{1}\left(X_{3} z\right) X_{2}\left(z_{s} \tau\right)+X_{4} z X_{2}\left(z_{\tau}\right)
$$

Because of the appearance of the higher-order commutator $X_{4}=\left[X_{1},\left[X_{1}, X_{2}\right]\right]$, formula (4.6) is different from the corresponding formula which one obtains in the 
case of the first Heisenberg group $\mathbb{H}^{1}$. Integrating (4.6) on $\Omega$ we find that

$$
\begin{aligned}
& -\int_{\Omega} X_{i} \frac{\partial H(z)}{\partial z_{, i j}} X_{j}\left(z_{s}\right) d g=-\frac{3}{2} \int_{\Omega}\left\{X_{2}(X-3 z) X_{1}\left(z_{s}\right)-X_{1}\left(X_{3} z\right) X_{2}\left(z_{\tau}\right)\right\} d g \\
& -\int_{\Omega} X_{4} z X_{2}\left(z_{\tau}\right) d g \\
& =-\frac{3}{2} \int_{\partial \Omega} X_{3} z\left\{X_{1}\left(z_{\tau}\right)\left\langle X_{2}, \nu\right\rangle-X_{2}\left(z_{\tau}\right)\left\langle X_{1}, \nu\right\rangle\right\} d \sigma \\
& +\frac{3}{2} \int_{\Omega} X_{3} z\left\{X_{2} X_{1}\left(z_{\tau}\right)-X_{1} X_{2}\left(z_{\tau}\right)\right\} d g-\int_{\Omega} X_{4} z X_{2}\left(z_{\tau}\right) d g \\
& =-\frac{3}{4} \frac{d}{d \tau} \int_{\Omega}\left(X_{3} z\right)^{2} d g-\int_{\Omega} X_{4} z X_{2}\left(z_{\tau}\right) d g,
\end{aligned}
$$

where in the second-to-last equality we have again used (4.1). Also, thanks to (2.14), the boundary integral vanishes since

$$
X_{1}\left(z_{\tau}\right)\left\langle X_{2}, \nu\right\rangle-X_{2}\left(z_{\tau}\right)\left\langle X_{1}, \nu\right\rangle=\left[\left\langle X_{1}, \nu\right\rangle\left\langle X_{2}, \nu\right\rangle-\left\langle X_{1}, \nu\right\rangle\left\langle X_{2}, \nu\right\rangle\right]\left|\nabla z_{\tau}\right| \text {. }
$$

Inserting (4.7) in (4.3) we find that

$$
\begin{aligned}
& \frac{d}{d \tau} \int_{\Omega}\left\{H(z)+\frac{3}{4}\left(X_{3} z\right)^{2}\right\} d g \\
& \quad=\int_{\partial \Omega} \frac{\partial H(z)}{\partial z_{, i j}} \nu_{X, i} \nu_{X, j}\left|\nabla z_{\tau}\right| d \sigma-\int_{\Omega} X_{4} z X_{2}\left(z_{\tau}\right) d g
\end{aligned}
$$

At this point it does not seem obvious that there is any monotonicity attached to (4.8). To disclose it, we integrate by parts the second term on the right-hand side obtaining

$$
\int_{\Omega} X_{4} z X_{2}\left(z_{\tau}\right) d g=\int_{\partial \Omega} z\left\langle X_{4}, \nu\right\rangle X_{2}\left(z_{\tau}\right) d \sigma-\int_{\Omega} z X_{4} X_{2}\left(z_{\tau}\right) d g .
$$

We also integrate by parts the following expression

$$
\begin{aligned}
\int_{\Omega} X_{2} z X_{4}\left(z_{\tau}\right) d g & =\int_{\partial \Omega} z\left\langle X_{2}, \nu\right\rangle X_{4}\left(z_{\tau}\right) d \sigma-\int_{\Omega} z X_{2} X_{4}\left(z_{\tau}\right) d g \\
& =\int_{\partial \Omega} z\left\langle X_{2}, \nu\right\rangle X_{4}\left(z_{\tau}\right) d \sigma-\int_{\Omega} z X_{4} X_{2}\left(z_{\tau}\right) d g,
\end{aligned}
$$

where in the last equality we have used $\left[X_{2}, X_{4}\right]=0$. Substituting (4.10) in (4.9) we find that

$$
\begin{aligned}
\int_{\Omega} X_{4} z X_{2}\left(z_{\tau}\right) d g & =\int_{\Omega} X_{2} z X_{4}\left(z_{\tau}\right) d g \\
& +\int_{\partial \Omega} z\left\{\left\langle X_{4}, \nu\right\rangle X_{2}\left(z_{\tau}\right)-\left\langle X_{2}, \nu\right\rangle X_{4}\left(z_{\tau}\right)\right\} d \sigma \\
& =\int_{\Omega} X_{2} z X_{4}\left(z_{\tau}\right) d g
\end{aligned}
$$

where again the boundary integral vanishes thanks to equation (2.14). Using (4.11) we finally recognize that

$$
\frac{d}{d \tau} \int_{\Omega} X_{4} z X_{2} z d g=2 \int_{\Omega} X_{4} z X_{2}\left(z_{\tau}\right) d g
$$


If we replace (4.12) in (4.8) we reach the conclusion

$$
\frac{d}{d \tau} \int_{\Omega}\left\{H(z)+\frac{3}{4}\left(X_{3} z\right)^{2}+\frac{1}{2}\left(X_{2} z\right)\left(X_{4} z\right)\right\} d g=\int_{\partial \Omega} \frac{\partial H(z)}{\partial z_{, i j}} \nu_{X, i} \nu_{X, j}\left|\nabla z_{\tau}\right| d \sigma .
$$

By the ellipticity of the horizontal Monge-Ampère equation on smooth weakly $H$-convex functions we conclude as before that $\frac{\partial H(z)}{\partial z, i j} \nu_{X, i} \nu_{X, j} \geq 0$, thus completing the proof.

Remark 4.2. It is interesting to observe that when the functions $u$ and $v$ depend only on the variables $(x, y)$ and $t$ in the first two layers, then $X_{4} u=X_{4} v=0$ and, at least formally (i.e., without taking into account the global boundary conditions), one obtains from Theorem 4.1 the statement of Theorem 1.1.

\section{Sub-ELliptiC CONES}

A crucial aspect of the theory of fully nonlinear equations is the possibility of comparing a convex function $u$, less than or equal to zero on the boundary of a ball, with a cone (or with its regularization) touching the graph of $u$ at its minimum point. Precisely, if $u$ is a convex function in $B=B(x, R) \subset \mathbb{R}^{n}$ such that $u \in C(\bar{B})$ and $u \leq 0$ on $\partial B$, and we consider the cone $\frac{u(x)}{R}(R-|y-x|)$, vanishing on $\partial B$ and touching $u$ at $y=x$, then one has for every $y \in B$

$$
u(y) \leq \frac{u(x)}{R}(R-|y-x|) .
$$

The purpose of this section is to establish a basic sub-elliptic counterpart of this property. We stress that the elementary Euclidean proof of (5.1) cannot be reproduced for weakly $H$-convex functions in a Carnot group, since the notion of weak $H$-convexity (2.8) only involves control on the horizontal plane, and one has to develop a different, more sophisticated approach.

Cones in Euclidean space are built on the Lipschitz function $\Gamma(x)=|x|$. Such a function has the property of providing the fundamental solution for the MongeAmpère equation. As we will see in Proposition 6.6. such a property breaks down for the sub-elliptic Monge-Ampère equation det $\operatorname{Hess}_{X}(u)=0$. Nonetheless, in any group of Heisenberg type the anisotropic gauge $N(g)$ does provide an explicit singular solution. One has in fact the following result, which is Theorem 10.9 in [DGN1.

Theorem 5.1. Let $\boldsymbol{G}$ be a group of Heisenberg type. Then the gauge $N(g)=$ $\left(|x(g)|^{4}+16|y(g)|^{2}\right)^{1 / 4}$ satisfies the equation $\operatorname{det}$ Hess $_{X} N=0$ in $\boldsymbol{G} \backslash\{0\}$.

Furthermore, one has the following basic information, which is Theorem 6.8 in DGN1.

Theorem 5.2. Let $\boldsymbol{G}$ be a group of Heisenberg type. Then the gauge $N(g)=$ $\left(|x(g)|^{4}+16|y(g)|^{2}\right)^{1 / 4}$ is weakly $H$-convex.

Theorems 5.1 and 5.2 strongly suggest that, at least for groups of Heisenberg type, one should build cones using the distance $d\left(g, g^{\prime}\right)=N\left(g^{-1} \circ g^{\prime}\right)$ associated with the gauge (it is not trivial that $d$ is an actual distance; for this fact, see $[\mathrm{Cy}]$ ). 
Definition 5.3. In a group of Heisenberg type $\boldsymbol{G}$ consider the gauge ball $B(0, R)=$ $\{g \in \boldsymbol{G} \mid N(g)<R\}$. The sub-elliptic cone based on $B(0, R)$ with height $h>0$ is defined by

$$
\Gamma_{H}(g)=\frac{h}{R}(N(g)-R) .
$$

We define the cone based on $B\left(g_{o}, R\right), g_{o} \in \boldsymbol{G}$, as $g \rightarrow \Gamma_{H}\left(g_{o}^{-1} g\right)$. The regularized cone based on $B(0, R)$ is given by

$$
\Gamma_{H, \epsilon}(g)=\frac{h}{R}\left(\left(N(g)^{4}+\epsilon^{4}\right)^{1 / 4}-\left(R^{4}+\epsilon^{4}\right)^{1 / 4}\right), \quad \epsilon>0 .
$$

We note explicitly that $\Gamma_{H}$ is only Lipschitz continuous with respect to the Carnot-Carathéodory metric of $\boldsymbol{G}$. Once Definition 5.3 is introduced, one faces the problem of establishing an appropriate analogue of (5.1). Unfortunately, an elementary direct approach does not seem available. On the other hand, Euclidean cones enjoy another basic property: they are singular solutions of the nonlinear operator $\Delta_{\infty} u=\sum_{i, j=1}^{n} D_{i j} u D_{i} u D_{j} u$ introduced by G. Aronsson in his classical papers [Ar1, Ar2 . Thereby, an indirect (and quite nonelementary) proof of (5.1) can be obtained by appealing to the fundamental comparison theorem for viscosity solutions of $\Delta_{\infty}$ due to R. Jensen [J].

This brings us to introduce the following strongly nonlinear operator. In a Carnot group $\boldsymbol{G}$ the $\infty$-sub-Laplacian is defined by

$$
\mathcal{L}_{\infty} u=\left\langle\operatorname{Hess}_{X}(u) X u, X u\right\rangle=\sum_{i, j=1}^{m} X_{i} X_{j} u X_{i} u X_{j} u .
$$

A classical solution is a function $u \in \Gamma^{2}(\boldsymbol{G})$ which solves $\mathcal{L}_{\infty} u=0$ in the classical sense. The following result will be crucial in the sequel; see Proposition 6.4 in DGN1.

Theorem 5.4. Let $\boldsymbol{G}$ be a group of Heisenberg type, with gauge $N(g)=$ $\left(|x(g)|^{4}+16|y(g)|^{2}\right)^{1 / 4}$. Then $\mathcal{L}_{\infty} N=0$, in the classical sense in $\boldsymbol{G} \backslash\{0\}$.

In the Heisenberg group Tom Bieske has introduced a notion of viscosity solution for (5.4) based on comparison with suitable quadratic polynomials weighted according to the grading of the Lie algebra (see $\mathrm{B}$ ), and also the subsequent developments due to Bieske and L. Capogna $[\mathrm{BC}$. Bieske's comparison theorem, and the recent generalization of such a result due to C. Wang [Wa1, will be important to us. We mention that, based on the results in [B], Lu, Manfredi and Stroffolini have defined cones in [LMS] by solving the Dirichlet problem for (5.4) on metric balls. However, such general perspective which has an obvious interest would presently be of no use for us. The reason being that to carry our program we have to make extensive explicit computations for the sub-elliptic cones, or better for their regularizations, of all the geometric quantities involved in the definition (1.7). In connection with viscosity solutions of $\mathcal{L}_{\infty}$ we will also need the following result.

Proposition 5.5. Given a Carnot group $\boldsymbol{G}$, let $u \in C(\boldsymbol{G})$ be a weakly $H$-convex function; then $u$ is a viscosity subsolution of $\mathcal{L}_{\infty}$.

Proof. After Proposition 10.7 in [DGN1] it was proved that in every Carnot group a continuous weakly $H$-convex function is a viscosity subsolution of the horizontal Monge-Ampère equation. This is trickier than proving the proposition, as one has 
to involve horizontal planes. A trivial modification of that argument allows us to reach the sought-for conclusion for the operator $\mathcal{L}_{\infty}$.

The next result is Theorem 1.6 in $\mathrm{B}$ for the Heisenberg group $\mathbb{H}^{n}$, and Theorem $\mathrm{C}$ in Wa1 for all Carnot groups. The notions of sub- and supersolutions of $\mathcal{L}_{\infty}$ are intended in the viscosity sense introduced in $[\mathrm{B}$.

Theorem 5.6. Given a Carnot group $\boldsymbol{G}$, let $\Omega \subset \boldsymbol{G}$ be a connected open set, $u$ an upper semicontinuous subsolution, and $v$ a lower semicontinuous supersolution in $\Omega$ of the $\infty$-sub-Laplacian $\mathcal{L}_{\infty}$. Suppose that for every $g_{o} \in \partial \Omega$ one has

$$
\limsup _{g \rightarrow g_{o}} u(g) \leq \liminf _{g \rightarrow g_{o}} v(g),
$$

where both sides are not $-\infty$ or $+\infty$ simultaneously; then $u \leq v$ in $\Omega$.

We now return to the main objective of this section.

Lemma 5.7. Let $\boldsymbol{G}$ be a group of Heisenberg type. For every $\epsilon>0$ the regularized cone $\Gamma_{H, \epsilon} \in C^{\infty}(\boldsymbol{G})$ is weakly $H$-convex.

Proof. The real-valued function $k(s)=\left(s^{4}+\epsilon^{4}\right)^{1 / 4}-\left(R^{4}+\epsilon^{4}\right)^{1 / 4}$ is nondecreasing and convex on the interval $s \geq 0$. One has in fact

$$
k^{\prime}(s)=s^{3}\left(s^{4}+\epsilon^{4}\right)^{1 / 4} \geq 0, \quad k^{\prime \prime}(s)=\frac{3 \epsilon^{4} s^{2}}{\left(s^{4}+\epsilon^{4}\right)^{7 / 4}} \geq 0 .
$$

Since $\Gamma_{H, \epsilon}=k(N)$, from Theorem 5.2 we infer the conclusion.

The next simple lemma will be important in the sequel.

Lemma 5.8. Let $\epsilon>0$ be fixed; then one has for every $g \in B(0, R)$

$$
\Gamma_{H}(g) \leq \Gamma_{H, \epsilon}(g) .
$$

Proof. Consider the real-valued function

$$
f(s)=\frac{h}{R}(s-R)-\frac{h}{R}\left[\left(s^{4}+\epsilon^{4}\right)^{1 / 4}-\left(R^{4}+\epsilon^{4}\right)^{1 / 4}\right], \quad 0 \leq s \leq R .
$$

Clearly, $f(R)=0$. Moreover,

$$
f^{\prime}(s)=\frac{h}{R} \frac{\left(s^{4}+\epsilon^{4}\right)^{3 / 4}-s^{3}}{\left(s^{4}+\epsilon^{4}\right)^{3 / 4}} \geq 0 .
$$

We infer $f(s) \leq 0$ for every $0 \leq s \leq R$. This proves the lemma.

With these ingredients in hand we can finally prove the following sub-elliptic version of (5.1).

Theorem 5.9. Let $\boldsymbol{G}$ be a group of Heisenberg type and let $u \in C(B)$ be a weakly $H$-convex function in $B=B(0, R)$ such that limsup $u \leq 0$.

Consider the cone (5.2), with $h=-u(0)$. This means that $\Gamma_{H}$ touches $u$ at $g=0$. One has

$$
u(g) \leq \Gamma_{H}(g), \quad \text { for every } g \in B .
$$

Combining (5.5) with Lemma 5.8, we also have

$$
u(g) \leq \Gamma_{H, \epsilon}(g), \quad \text { for every } g \in B .
$$


Proof. To prove (5.5) consider the connected open set $\Omega=B \backslash\{0\}$. The weakconvexity of $u$, and Proposition 5.5. imply that $u$ is a viscosity subsolution of $\mathcal{L}_{\infty}$ in $\Omega$. On the other hand, by Theorem 5.4 we have $\mathcal{L}_{\infty} \Gamma_{H}=0$ in $\Omega$ in the classical sense. Since

$$
\limsup _{\partial B} u \leq 0=\lim _{\partial B} \Gamma_{H}
$$

and since moreover

$$
\lim _{\delta \rightarrow 0} \sup _{\partial B(0, \delta)}\left|\Gamma_{H}-u\right|=0
$$

by Theorem 5.6 we conclude that (5.5) holds.

\section{Monge-Ampère MeAsures of SUb-ELLIPTIC CONES}

If $\Gamma(x)=\frac{h}{R}(|x|-R)$ is a standard cone in $\mathbb{R}^{n}$ based on the ball $B(0, R)$, and if $\Gamma_{\epsilon}(x)=\frac{h}{R}\left(\left(|x|^{2}+\epsilon^{2}\right)^{1 / 2}-\left(R^{2}+\epsilon^{2}\right)^{1 / 2}\right)$ indicates its regularization, then

$$
\lim _{\epsilon \rightarrow 0} \int_{B(0, R)} \operatorname{det}\left(D^{2} \Gamma_{\epsilon}\right) d x=\left|B\left(0, \frac{h}{R}\right)\right| .
$$

The equation (6.1) can be reformulated by saying that the Alexandrov (or Monge-Ampère) measure of the cone $\Gamma$ is given by the formula

$$
M(\Gamma)=\left|B\left(0, \frac{h}{R}\right)\right| \delta,
$$

which plays a critical role in the classical theory of convex functions. The aim of this section is to establish an ad hoc version of (6.1) which is important in the development of fully nonlinear sub-elliptic equations. To accomplish this we need to develop various explicit calculations, which also have an interest in their own right. Our first goal is to compute the action of the fully nonlinear operator introduced in (1.7) on the regularized cones in (5.3). A direct calculation is quite complicated, so we approach the problem from a general point of view, which eventually leads to a more elegant and simpler solution. We let $u$ be a function in $\Gamma^{2}\left(\mathbb{H}^{2}\right)$ given in the form

$$
u(z, t)=f(|z|, t)=f(r, t), \quad r=|z| .
$$

We are interested in computing the determinant of the horizontal Hessian of such a function. This is given in the following result.

Proposition 6.1. Let $u$ be as in (6.3); then we have

$$
\operatorname{det} \operatorname{Hess}_{X}(u)=\left(\frac{f_{r}}{r}\right)^{2}\left\{\frac{r^{2}}{4}\left[f_{r r} f_{t t}-f_{r t}^{2}\right]+\frac{1}{r} f_{r r} f_{r}\right\} .
$$

Proof. In the sequel it will be convenient to introduce the following notation:

$$
F=f_{r r}-\frac{f_{r}}{r} .
$$


With such agreement we obtain for the symmetrized second derivatives

$$
\begin{aligned}
& u_{, 11}=\frac{x_{1}^{2}}{r^{2}} F-\frac{x_{1} y_{1}}{r} f_{r t}+\frac{y_{1}^{2}}{4} f_{t t}+\frac{f_{r}}{r}, \\
& u_{, 12}=\frac{x_{1} x_{2}}{r^{2}} F-\frac{x_{1} y_{2}+x_{2} y_{1}}{2 r} f_{r t}+\frac{y_{1} y_{2}}{4} f_{t t}, \\
& u_{, 13}=\frac{x_{1} y_{1}}{r^{2}} F+\frac{x_{1}^{2}-y_{1}^{2}}{2 r} f_{r t}-\frac{x_{1} y_{1}}{4} f_{t t}, \\
& u_{, 14}=\frac{x_{1} y_{2}}{r^{2}} F+\frac{x_{1} x_{2}-y_{1} y_{2}}{2 r} f_{r t}-\frac{x_{2} y_{1}}{4} f_{t t}, \\
& u_{, 21}=u_{, 12}, \\
& u_{, 22}=\frac{x_{2}^{2}}{r^{2}} F-\frac{x_{2} y_{2}}{r} f_{r t}+\frac{y_{2}^{2}}{4} f_{t t}+\frac{f_{r}}{r}, \\
& u_{, 23}=\frac{x_{2} y_{1}}{r^{2}} F+\frac{x_{1} x_{2}-y_{1} y_{2}}{2 r} f_{r t}-\frac{x_{1} y_{2}}{4} f_{t t}, \\
& u_{, 24}=\frac{x_{2} y_{2}}{r^{2}} F+\frac{x_{2}^{2}-y_{2}^{2}}{2 r} f_{r t}-\frac{x_{2} y_{2}}{4} f_{t t}, \\
& u_{, 31}=u_{, 13}, \\
& u_{, 32}=u_{, 23}, \\
& u_{, 33}=\frac{y_{1}^{2}}{r^{2}} F+\frac{x_{1} y_{1}}{r} f_{r t}+\frac{x_{1}^{2}}{4} f_{t t}+\frac{f_{r}}{r}, \\
& u_{, 34}=\frac{y_{1} y_{2}}{r^{2}} F+\frac{x_{2} y_{1}+x_{1} y_{2}}{2 r} f_{r t}+\frac{x_{1} x_{2}}{4} f_{t t}, \\
& u_{, 41}=u_{, 14}, \\
& u_{, 42}=u_{, 24}, \\
& u_{, 43}=u_{, 34}, \\
& u_{, 44}=\frac{y_{2}^{2}}{r^{2}} F+\frac{x_{2} y_{2}}{r} f_{r t}+\frac{x_{2}^{2}}{4} f_{t t}+\frac{f_{r}}{r} .
\end{aligned}
$$

To compute det $\operatorname{Hess}_{X}(u)$ we exploit its invariance with respect to the orthogonal group in the first layer $\mathbb{R}^{4} \times\{0\}$ of $\mathbb{H}^{2}$. Accordingly, it suffices to compute such a determinant at the point $g=(z, t)$, with $z=(0,|z|, 0,0)$. Recalling that $r=|z|$, we find at this point $g$, from (6.5) and from (6.6)-(6.9),

$$
\left(\begin{array}{cccc}
\frac{f_{r}}{r} & 0 & 0 & 0 \\
0 & f_{r r} & 0 & \frac{r}{2} f_{r t} \\
0 & 0 & \frac{f_{r}}{r} & 0 \\
0 & \frac{r}{2} f_{r t} & 0 & \frac{f_{r}}{r}+\frac{r^{2}}{4} f_{t t}
\end{array}\right) .
$$

From (6.10) we easily obtain (6.4).

Our next task is to compute the nonlinear quantity $A_{3}^{1}(u)+A_{4}^{2}(u)$ in the second term of the sub-elliptic Monge-Ampère operator in definition (1.7), when $u$ is a function as in (6.3).

Proposition 6.2. Let $u$ be as in (6.3); then we have

$$
A_{3}^{1}(u)+A_{4}^{2}(u)=\frac{r^{2}}{4}\left[f_{r r} f_{t t}-f_{r t}^{2}\right]+\frac{1}{r} f_{r r} f_{r}+\left(\frac{f_{r}}{r}\right)^{2} .
$$


Proof. We recall that

$$
A_{3}^{1}(u)+A_{4}^{2}(u)=u_{, 11} u_{, 33}-u_{, 13}^{2}+u_{, 22} u_{, 44}-u_{, 24}^{2}+2\left(u_{, 12} u_{, 34}-u_{, 14} u_{, 23}\right) .
$$

Using (6.6)-(6.9), after a lengthy computation we find that

$$
\begin{aligned}
& u_{, 11} u_{, 33}-u_{, 13}^{2}=\frac{\left|z_{1}\right|^{4}}{4 r^{2}}\left(F f_{t t}-f_{r t}^{2}\right)+\frac{\left|z_{1}\right|^{2}}{r^{3}} F f_{r}+\frac{\left|z_{1}\right|^{2}}{4 r} f_{t t} f_{r}+\left(\frac{f_{r}}{r}\right)^{2}, \\
& u_{, 22} u_{, 44}-u_{, 24}^{2}=\frac{\left|z_{2}\right|^{4}}{4 r^{2}}\left(F f_{t t}-f_{r t}^{2}\right)+\frac{\left|z_{2}\right|^{2}}{r^{3}} F f_{r}+\frac{\left|z_{2}\right|^{2}}{4 r} f_{t t} f_{r}+\left(\frac{f_{r}}{r}\right)^{2}, \\
& u_{, 12} u_{, 34}-u_{, 14} u_{, 23}=\frac{\left|z_{1}\right|^{2}\left|z_{2}\right|^{2}}{4 r^{2}}\left(F f_{t t}-f_{r t}^{2}\right) .
\end{aligned}
$$

In (6.13) we have identified the complex number $z_{j}=x_{j}+i y_{j}, j=1,2$, with the point $\left(x_{j}, y_{j}\right) \in \mathbb{R}^{2}$, so that $|z|^{2}=\left|z_{1}\right|^{2}+\left|z_{2}\right|^{2}$, with $\left|z_{j}\right|^{2}=x_{j}^{2}+y_{j}^{2}$. Substitution of (6.13) in (6.12) yields the conclusion (6.11).

Using Propositions 6.1 and 6.2 we next turn to the computation of the operator in (1.7) acting on the regularized cone (5.3).

Proposition 6.3. In $\mathbb{H}^{2}$ we have

$$
\operatorname{det} \operatorname{Hess}_{X}\left(\Gamma_{H, \epsilon}\right)=\frac{h^{4}}{R^{4}} \frac{9 \epsilon^{4}|z|^{8}}{\left(N(g)^{4}+\epsilon^{4}\right)^{4}} .
$$

Proof. We write $\Gamma_{H, \epsilon}$ in the form (6.3), with

$$
f(r, t)=\frac{h}{R}\left\{\left(r^{4}+16 t^{2}+\epsilon^{4}\right)^{1 / 4}-\left(R^{4}+\epsilon^{4}\right)^{1 / 4}\right\} .
$$

Simple calculations give

$$
\begin{gathered}
f_{r}=\frac{h}{R} r^{3}\left(r^{4}+16 t^{2}+\epsilon^{4}\right)^{-3 / 4}, \\
f_{r r}=3 \frac{h}{R} r^{2}\left(16 t^{2}+\epsilon^{4}\right)\left(r^{4}+16 t^{2}+\epsilon^{4}\right)^{-7 / 4}, \\
f_{r t}=-24 \frac{h}{R} r^{3} t\left(r^{4}+16 t^{2}+\epsilon^{4}\right)^{-7 / 4}, \\
f_{t}=8 \frac{h}{R} t\left(r^{4}+16 t^{2}+\epsilon^{4}\right)^{-3 / 4}, \\
f_{t t}=8 \frac{h}{R}\left(r^{4}-8 t^{2}+\epsilon^{4}\right)\left(r^{4}+16 t^{2}+\epsilon^{4}\right)^{-7 / 4} .
\end{gathered}
$$

For later purposes it will be expedient to record the following quantity, which is obtained from (6.14)-(6.18) in (6.4), by elementary algebraic manipulations:

$$
\frac{r^{2}}{4}\left[f_{r r} f_{t t}-f_{r t}^{2}\right]+\frac{1}{r} f_{r r} f_{r}=9 \frac{h^{2}}{R^{2}} \epsilon^{4} r^{4}\left(r^{4}+16 t^{2}+\epsilon^{4}\right)^{-10 / 4} .
$$

From (6.14), (6.19), (6.4) it is now easy to obtain the sought-for conclusion.

Remark 6.4. We emphasize that letting $\epsilon \rightarrow 0$ in Proposition 6.3 we obtain

$$
\operatorname{det} \operatorname{Hess}_{X}\left(\Gamma_{H}\right)=0 \quad \text { in } \mathbb{H}^{2} \backslash\{0\} .
$$

This is, in fact, just a reformulation of the fundamental property of the gauge of being a singular solution of the horizontal Monge-Ampère equation; see Theorem 5.1 . 
Proposition 6.5. In $\mathbb{H}^{2}$ one has

$$
A_{3}^{1}\left(\Gamma_{H, \epsilon}\right)+A_{4}^{2}\left(\Gamma_{H, \epsilon}\right)=\frac{h^{2}}{R^{2}} \frac{|z|^{4}}{\left(N(g)^{4}+\epsilon^{4}\right)^{10 / 4}}\left(N(g)^{4}+10 \epsilon^{4}\right) .
$$

Proof. It is an immediate consequence of (6.11) in Proposition 6.2, of (6.14), and of (6.19).

The next result is in sharp contrast with (6.1), and also proves that the analogue of (6.2) cannot possibly hold for $\mathbb{H}^{n}$.

Proposition 6.6. In $\mathbb{H}^{n}$ one has

$$
\lim _{\epsilon \rightarrow 0} \int_{B(0, R)} \operatorname{det} \operatorname{Hess}_{X}\left(\Gamma_{H, \epsilon}\right) d g=0 .
$$

Proof. We only give the proof for $\mathbb{H}^{2}$, since this is the case in which we will apply the result. Applying Proposition 6.3 we find

$$
\begin{aligned}
\int_{B(0, R)} \operatorname{det} \operatorname{Hess}_{X}\left(\Gamma_{H, \epsilon}\right) d g & =\frac{9 \epsilon^{4} h^{4}}{R^{4}} \int_{B(0, R)} \frac{|z|^{8}}{\left(N(g)^{4}+\epsilon^{4}\right)^{16 / 4}} d g \\
& \leq \frac{9 \epsilon^{4} h^{4}}{R^{4}} \int_{B(0, R)} \frac{\Phi(g)}{\left(N(g)^{4}+\epsilon^{4}\right)^{2}} d g
\end{aligned}
$$

where we have let

$$
\Phi(g)=\frac{|z|^{8}}{N(g)^{8}} .
$$

Note that $0 \leq \Phi \leq 1$, and that moreover $\Phi$ is homogeneous of degree 0 with respect to the anisotropic group dilations, so $\Phi$ is concentrated on the unit gauge sphere $\partial B(0,1)$. By Proposition 1.15 in [FS2, given a Carnot group $\boldsymbol{G}$ with gauge $N$, there exists a unique Radon measure $\mu$ on $\partial B(0,1)$ such that if $u \in L^{1}(B(0, R))$ one has

$$
\int_{B(0, R)} u(g) d g=\int_{0}^{R} \int_{\partial B(0,1)} u\left(\delta_{\rho} \omega_{g}\right) d \mu(g) \rho^{Q} \frac{d \rho}{\rho},
$$

where $Q$ indicates the homogeneous dimension of $\boldsymbol{G}$, and we have denoted by $\omega_{g}=$ $\delta_{N(g)^{-1}} g$ the anisotropic projection of $g$ onto $B(0,1)$. Applying this formula to (6.20), and keeping in mind that in $\mathbb{H}^{2}$ one has $Q=6$, we find

$$
\int_{B(0, R)} \frac{\Phi(g)}{\left(N(g)^{4}+\epsilon^{4}\right)^{2}} d g=\omega \int_{0}^{R} \frac{\rho^{6}}{\left(\rho^{4}+\epsilon^{4}\right)^{2}} \frac{d \rho}{\rho},
$$

where we have let

$$
\omega=\int_{\partial B(0,1)} \Phi(g) d \mu(g)>0 .
$$

By standard calculus techniques we find

$$
\int_{0}^{R} \frac{\rho^{6}}{\left(\rho^{4}+\epsilon^{4}\right)^{2}} \frac{d \rho}{\rho}=\frac{1}{4 \epsilon^{2}}\left[\tan ^{-1}\left(\frac{R^{2}}{\epsilon^{2}}\right)-\frac{\epsilon^{2} R 62}{R^{4}+\epsilon^{4}}\right] .
$$

Combining the latter equation with (6.20), (6.21), one obtains

$$
\int_{B(0, R)} \operatorname{det} \operatorname{Hess}_{X}\left(\Gamma_{H, \epsilon}\right) d g=\frac{9 \omega h^{4}}{4 R^{4}}\left[\tan ^{-1}\left(\frac{R^{2}}{\epsilon^{2}}\right)-\frac{\epsilon^{2} R^{2}}{R^{4}+\epsilon^{4}}\right] \epsilon^{2} \longrightarrow 0,
$$

as $\epsilon \rightarrow 0$. This completes the proof. 
Remark 6.7. In Section 9 we will discuss the impossibility of the estimate (9.4). Proposition 6.6 allows us to give another proof of such a negative phenomenon. Consider in fact, for fixed $h, R>0$, the $C^{\infty}$ functions $\Gamma_{H, \epsilon}$, which locally uniformly converge to the sub-elliptic cone $\Gamma_{H}(g)=\frac{h}{R}[R-N(g)]$. If the inequality (9.4) were true, with a constant $C>0$ independent of $u$, then keeping in mind that $\Gamma_{H, \epsilon}=0$ on $\partial B((0, R)$, we would obtain on $\Omega=B(0, R)$

$$
\sup _{\Omega}\left|\Gamma_{H, \epsilon}\right| \leq C \int_{\Omega} \operatorname{det} \operatorname{Hess}_{X}\left(\Gamma_{H, \epsilon}\right) d g .
$$

Simple considerations allow us to conclude that

$$
\sup _{\Omega}\left|\Gamma_{H, \epsilon}\right|=\left|\Gamma_{H, \epsilon}(0)\right|=\frac{h}{R}\left[\left(R^{4}-\epsilon^{4}\right)^{1 / 4}-\epsilon\right] \longrightarrow h, \quad \text { as } \quad \epsilon \rightarrow 0 .
$$

Substituting this information in (6.22) and using Proposition 6.6, after letting $\epsilon \rightarrow 0$ we reach the contradiction $h \leq 0$. This proves that (9.4) cannot possibly hold.

We are now in a position to establish a sub-elliptic counterpart of (6.1). As we have seen from Proposition 6.6 there is no contribution from the horizontal Hessian of the regularized cone. However, we will see that, due to the presence of the commutator, the second and third terms in the integral in Theorem 2.1 contribute in equal manner to the Monge-Ampère measure.

Theorem 6.8. Let $\Gamma_{H, \epsilon}$ be the regularized cone defined in (5.3). Then there exists an absolute constant $\omega>0$ such that for any $h, R>0$ one has in $\mathbb{H}^{2}$

$$
\lim _{\epsilon \rightarrow 0} \int_{B(0, R)} \mathcal{S}_{m a}\left(\Gamma_{H, \epsilon}\right) d g=\omega \frac{h^{4}}{R^{2}},
$$

where $\mathcal{S}_{m a}\left(\Gamma_{H, \epsilon}\right)$ is as in (1.7).

Proof. Thanks to Proposition 6.6 we only need to evaluate the limit of the integral of the second and third terms in the expression of $S_{m a}\left(\Gamma_{H, \epsilon}\right)$; see (1.7). To compute the limit of the second term we combine (6.17) with Proposition 6.5, obtaining

$$
\begin{aligned}
& \int_{B(0, R)}\left[A_{3}^{1}\left(\Gamma_{H, \epsilon}\right)+A_{4}^{2}\left(\Gamma_{H, \epsilon}\right)\right]\left(T \Gamma_{H, \epsilon}\right)^{2} d g \\
& =4 \frac{h^{4}}{R^{4}} \int_{B(0, R)} \frac{16 t^{2}|z|^{4}\left(N(g)^{4}+10 \epsilon^{4}\right)}{\left(N(g)^{4}+\epsilon^{4}\right)^{4}} d g \\
& =4 \frac{h^{4}}{R^{4}} \int_{B(0, R)} \frac{16 t^{2}|z|^{4} N(g)^{4}}{\left(N(g)^{4}+\epsilon^{4}\right)^{4}} d g+40 \epsilon^{4} \frac{h^{4}}{R^{4}} \int_{B(0, R)} \frac{16 t^{2}|z|^{4}}{\left(N(g)^{4}+\epsilon^{4}\right)^{4}} d g .
\end{aligned}
$$

By computations similar to those in the proof of Proposition 6.6, we find that

$$
\lim _{\epsilon \rightarrow 0} \epsilon^{4} \frac{h^{4}}{R^{4}} \int_{B(0, R)} \frac{16 t^{2}|z|^{4}}{\left(N(g)^{4}+\epsilon^{4}\right)^{4}} d g=0 .
$$

To evaluate the first integral on the right-hand side of (6.24) instead, we observe that for every fized $g=(z, t) \in \mathbb{H}^{2}$

$$
\lim _{\epsilon \rightarrow 0} \frac{16 t^{2}|z|^{4} N(g)^{4}}{\left(N(g)^{4}+\epsilon^{4}\right)^{4}}=\frac{\Theta(g)}{N(g)^{4}},
$$

where the nonnegative function

$$
\Theta(g) \stackrel{\text { def }}{=} \frac{16 t^{2}|z|^{4}}{N(g)^{8}}
$$


is homogeneous of degree zero with respect to the anisotropic group dilations, and has $L^{\infty}$ norm $\leq 1$. Since in $\mathbb{H}^{2}$ the homogeneous dimension is $Q=6$, and since $N^{-p} \in L_{l o c}^{1}\left(\mathbb{H}^{2}\right)$ provided that $p<Q$, we see that

$$
\frac{\Theta}{N^{4}} \in L^{1}(B(0, R))
$$

and therefore by Lebesgue dominated convergence we conclude that

$$
\lim _{\epsilon \rightarrow 0} 4 \frac{h^{4}}{R^{4}} \int_{B(0, R)} \frac{16 t^{2}|z|^{4} N(g)^{4}}{\left(N(g)^{4}+\epsilon^{4}\right)^{4}} d g=4 \frac{h^{4}}{R^{4}} \int_{B(0, R)} \frac{\Theta(g)}{N(g)^{4}} d g .
$$

A simple rescaling argument now gives

$$
\int_{B(0, R)} \frac{\Theta(g)}{N(g)^{4}} d g=R^{6} \int_{B(0,1)} \frac{\Theta\left(\delta_{R} g\right)}{N\left(\delta_{R} g\right)^{4}} d g=\omega_{1} R^{2}
$$

where we have denoted

$$
\omega_{1}=\int_{B(0,1)} \frac{\Theta(g)}{N(g)^{4}} d g>0 .
$$

Substituting (6.25)-(6.27) in (6.24), we reach the conclusion

$$
\lim _{\epsilon \rightarrow 0} \int_{B(0, R)}\left[A_{3}^{1}\left(u_{\epsilon}\right)+A_{4}^{2}\left(u_{\epsilon}\right)\right]\left(T u_{\epsilon}\right)^{2} d g=4 \omega_{1} \frac{h^{4}}{R^{2}} .
$$

We last turn to the third addend in (6.23). For this term considerations analogous to those which led to (6.28) permit us to prove that

$$
\lim _{\epsilon \rightarrow 0} \int_{B(0, R)}\left(T u_{\epsilon}\right)^{4} d g=16 \omega_{2} \frac{h^{4}}{R^{2}},
$$

where we have set

$$
\omega_{2}=\int_{B(0,1)} \frac{\Psi(g)}{N(g)^{4}} d g>0, \quad \Psi(g) \stackrel{\text { def }}{=}\left(\frac{16 t^{2}}{N(g)^{4}}\right)^{2} .
$$

Finally, combining Proposition 6.6 with (6.28) and (6.29), we conclude that (6.23) holds with

$$
\omega=3 \omega_{1}+5 \omega_{2}
$$

\section{Estimates from above of the generalized Monge-Ampère measure AND THE THEOREM OF Busemann-Feller-Alexandrov}

Our primary objective in this section is to obtain a local control from above of the fully nonlinear operator appearing in Theorem 2.1 in terms of the oscillation of the function $u$ with the purpose of establishing a delicate generalization of the classical theorem of Busemann-Feller-Alexandrov. Here, we adapt a beautiful idea from the paper by Trudinger and Wang [TW]. To establish our first main result, Theorem [7.1, we will need to provide a suitable smooth weakly $H$-convex barrier to insert in Theorem 2.1. The regularized sub-elliptic cones (or, equivalently an appropriate power of the latter) will provide the appropriate candidates. 
Theorem 7.1. Let $u \in \Gamma^{3}(\Omega)$ be weakly $H$-convex in $\Omega \subset \mathbb{H}^{n}, n=1,2$. For any $D \subset \subset \Omega$ we have for some constant $C>0$ depending on $\Omega, D$ and $n$,

$$
\int_{D} \mathcal{S}_{m a}(u) d g \leq C(\underset{\Omega}{\operatorname{osc} u})^{2 n},
$$

where $\mathcal{S}_{m a}(u)$ is the generalized Monge-Ampère operator in (1.7).

Proof. We only treat the case $n=2$, since the case $n=1$ is easier, as it does not require to use Theorem 3.2. Consider a gauge ball $B=B\left(g_{o}, R\right) \subset \Omega$, and without loss of generality we assume that $g_{o}=0$, the group identity. By considering the function $v=u-\sup _{B} u-\delta$ instead of $u$, we can assume that $v \leq-\delta$ in $\bar{B}$, for some $\delta>0$. If we set $m_{o}=\inf _{B} v<0$, we next introduce the function

$$
\psi(g)=3 \frac{\left|m_{o}\right|}{R}\left(\left(N(g)^{4}+\epsilon^{4}\right)^{1 / 4}-\left(R^{4}+\epsilon^{4}\right)^{1 / 4}\right)=3 \Gamma_{H, \epsilon},
$$

where $\Gamma_{H, \epsilon}$ is the regularized sub-elliptic cone based on $B$ with height $h=\left|m_{o}\right|$ introduced in (5.3). From Lemma 5.7 we know that the function $\psi$ is $C^{\infty}$ and weakly $H$-convex. Furthermore, we have

$$
\psi(0)=-3 \frac{\left|m_{o}\right|}{R}\left[\left(R^{4}+\epsilon\right)^{1 / 4}-\epsilon\right]<2 m_{o},
$$

provided that $\epsilon<R / 3$. We apply Theorem 2.1 to $v$ and $\psi$ on the open set $\tilde{B}=$ $\{g \in \Omega \mid \psi(g)<v(g)\}$, obtaining

$$
\begin{aligned}
\int_{\tilde{B}} \mathcal{S}_{m a}(v) d g & \leq \int_{\tilde{B}} \mathcal{S}_{m a}(\psi) d g \\
& \leq \int_{B} \mathcal{S}_{m a}(\psi) d g
\end{aligned}
$$

where in the last inequality we have used Theorem 3.2, which gives $\mathcal{S}_{m a}(\psi) \geq 0$.

We next observe that we trivially have

$$
\left\{g \in \Omega \mid \psi(g)<m_{o}\right\} \subset \tilde{B} .
$$

This being said, we now claim that there exists an absolute constant $\sigma \in(0,1)$, independent of $v$, such that

$$
B(0, \sigma R) \subset\left\{g \in \Omega \mid \psi(g)<m_{o}\right\} .
$$

The proof of (7.4) easily follows from the definition of $\psi$, provided that we choose $\sigma<\left[(2 / 3)^{4}-(1 / 4)^{4}\right]^{1 / 4}$. Using (7.4), (7.3), we can now appeal to Theorems 2.2 and 3.2 to obtain

$$
\int_{B(0, \sigma R)} \mathcal{S}_{m a}(v) d g \leq \int_{\tilde{B}} \mathcal{S}_{m a}(v) d g .
$$

Combining (7.5) with (7.2), we conclude that

$$
\int_{B(0, \sigma R)} \mathcal{S}_{m a}(v) d g \leq \int_{B} \mathcal{S}_{m a}(\psi) d g
$$

At this point, we recall the expression (7.1) of the function on the right-hand side of (7.6). Letting $\epsilon \rightarrow 0$, and invoking Theorem 6.8, we obtain

$$
\int_{B(0, \sigma R)} \mathcal{S}_{m a}(v) d g \leq \frac{3^{4} \omega}{R^{2}}\left|m_{o}\right|^{4}
$$


Finally, we let $\delta \rightarrow 0$ to reach the conclusion

$$
\int_{B(0, \sigma R)} \mathcal{S}_{m a}(u) d g \leq \frac{3^{4} \omega}{R^{2}}\left(\operatorname{osc}_{B} u\right)^{4} .
$$

To complete the proof, we simply cover $D \subset \subset \Omega$ with a finite number of balls $B\left(g_{j}, \sigma R\right)$, and apply (7.7) to each of these balls.

In the sequel we will need the following interesting result due to Balogh and Rickly (see Proposition 3.4 and Theorem 1.2 in $[\mathrm{BR}]$ ), and also the proof of Theorem 11.6 in DGN1].

Theorem 7.2. Let $u: \mathbb{H}^{n} \rightarrow \mathbb{R}$ be a weakly $H$-convex function; then $u$ is locally bounded and, in fact, continuous.

We now present an important consequence of Theorem 7.1, namely that the commutator of a weakly $H$-convex function is locally in $L_{l o c}^{2}$.

Theorem 7.3. Let $u$ be weakly $H$-convex in $\Omega \subset \mathbb{H}^{n}, n=1,2$. For any $D \subset \subset \Omega$ we have $T u \in L^{2 n}(D)$, where $T u$ denotes the distributional derivative of $u$.

Proof. Thanks to Theorem 7.2 we can assume that $u \in C(\Omega)$. Fix $D \subset \subset D^{\prime} \subset \subset \Omega$. Let $K \in C_{o}^{\infty}\left(\mathbb{H}^{n}\right)$ be such that $K \geq 0$, supp $K \subseteq \bar{B}(0,1), \int_{\mathbb{H}^{n}} K(g) d g=1$, and let $K_{\epsilon}(g)=\epsilon^{-Q} K\left(\delta_{\epsilon^{-1}} g\right)$ be the approximation to the identity associated with $K$. By Remark 5.9 in DGN1, for sufficiently small $\epsilon$, depending on $\operatorname{dist}\left(D^{\prime}, \Omega\right)$, the function $u_{\epsilon}=K_{\epsilon} \star u$ is weakly $H$-convex in $D^{\prime}$ and $C^{\infty}$. Furthermore, since $u_{\epsilon} \rightarrow u$ uniformly on compact subsets of $\Omega$, we clearly have

$$
\underset{D^{\prime}}{\operatorname{osc}} u_{\epsilon} \leq C \underset{\Omega}{\operatorname{osc}} u
$$

for some constant $C>0$ depending only on $\operatorname{dist}\left(D^{\prime}, \Omega\right)$, but not on $\epsilon$. From the latter inequality, and from Theorem 7.1, we find that

$$
\int_{D} \mathcal{S}_{m a}\left(u_{\epsilon}\right) d g \leq C\left(\underset{\Omega^{\prime}}{\operatorname{osc} u}\right)^{2 n}=C\left(\Omega, \Omega^{\prime}, n, u\right)<\infty .
$$

Invoking Theorem 2.2 and Theorem 3.2 , we conclude from (7.8) that

$$
\int_{D}\left(T u_{\epsilon}\right)^{2 n} d g \leq C\left(\Omega, \Omega^{\prime}, n, u\right) .
$$

In particular, (7.9) says that $\left\|T u_{\epsilon}\right\|_{L^{2 n}(D)} \leq C\left(\Omega, \Omega^{\prime}, n, u\right)$, and therefore there exists $v \in L^{2 n}(D)$ such that $T u_{\epsilon} \rightarrow v$. Denoting by $T u$ the distributional derivative of $u$ along $T$, one easily recognizes that $T u=v \in L^{2 n}(D)$. This proves the theorem.

We now recall a basic result, which is Theorem 8.1 in [DGN1]; see also [LMS].

Theorem 7.4. Let $\boldsymbol{G}$ be a Carnot group $\boldsymbol{G}$ and consider a weakly $H$-convex function $u \in L_{\text {loc }}^{1}(\boldsymbol{G})$. For $i, j=1, \ldots, m$, there exist signed Radon measures $\nu_{H}^{i j}=\nu_{H}^{j i}$ such that for every $\phi \in C_{o}^{\infty}(\boldsymbol{G})$ one has

$$
\int_{G} u(g) \phi_{, i j}(g) d g=\int_{G} u(g) \frac{X_{i} X_{j} \phi(g)+X_{j} X_{i} \phi(g)}{2} d g=\int_{G} \phi(g) d \nu_{H}^{i j}(g) .
$$

In addition, the measures $\nu_{H}^{i i}$ are nonnegative.

With Theorem 7.4 we can establish the following important consequence of Theorem 7.3 , 
Theorem 7.5. Let $u$ be weakly $H$-convex in $\Omega \subset \mathbb{H}^{n}, n=1,2$. Then the nonsymmetrized dsitributional second derivatives $X_{i} X_{j} u, i, j=1, \ldots, 2 n$, are signed Radon measures.

Proof. It is enough to observe that

$$
X_{i} X_{j} u=u_{, i j}+\frac{1}{2}\left[X_{i}, X_{j}\right] u \quad \text { in } \quad \mathcal{D}^{\prime}(\Omega) .
$$

Since $\left[X_{i}, X_{j}\right] u=\delta_{i j} T u$, from Theorem 7.3 we conclude that $\left[X_{i}, X_{j}\right] u \in L_{l o c}^{2 n}(\Omega)$, hence in particular all first commutators are Radon measures. The conclusion thus follows from the above identity and from Theorem 7.4 .

We next recall Theorem 9.1 from [DGN1].

Theorem 7.6. Let $\boldsymbol{G}$ be a Carnot group and $u \in C(\boldsymbol{G})$ be a weakly $H$-convex function. Then u is locally Lipschitz continuous with respect to the Carnot-Carathéodory distance. Furthermore, there exists a constant $L=L(\boldsymbol{G})>0$ such that for every $g_{o} \in \boldsymbol{G}$ and every $R>0$

$$
\begin{gathered}
\|X u\|_{L^{\infty}\left(B\left(g_{o}, R\right)\right)} \leq L\|u\|_{L^{\infty}\left(B\left(g_{o}, 3 R\right)\right)}, \\
\left|u(g)-u\left(g^{\prime}\right)\right| \leq L\|u\|_{L^{\infty}\left(B\left(g_{o}, 3 R\right)\right)} d\left(g, g^{\prime}\right), \quad g, g^{\prime} \in B\left(g_{o}, R\right) .
\end{gathered}
$$

In particular, there exist no weakly $H$-convex functions in $C(\boldsymbol{G})$, other than the constants.

To state our next result we recall the notion of horizontal bounded variation introduced in $\mathrm{CDG}$; see also $\mathrm{GN}$.

Let $\Omega \subset \boldsymbol{G}$ be an open set in a Carnot group $\boldsymbol{G}$, and $u \in L_{l o c}^{1}(\Omega)$. Denote by $\zeta=\sum_{i=1}^{m} \zeta_{i} X_{i}$ an element of $C_{o}^{1}(\Omega ; H \boldsymbol{G})$. Let

$$
\mathcal{F}_{H}(\Omega)=\left\{\zeta \in C_{o}^{1}(\Omega ; H \boldsymbol{G}) \mid\|\zeta\|_{\infty} \leq 1\right\} .
$$

The $H$-variation of $u$ in $\Omega$ is defined as follows:

$$
\operatorname{Var}_{H}(u ; \Omega)=\sup _{\zeta \in \mathcal{F}_{H}(\Omega)} \int_{\Omega} u \sum_{i=1}^{m} X_{i} \zeta_{i} d g
$$

A function $u \in L^{1}(\Omega)$ is called of bounded H-variation if $\operatorname{Var}_{H}(u ; \Omega)<\infty$. In such a case, we write $u \in B V_{H}(\Omega)$, and the collection of all such functions becomes a Banach space when endowed with the norm

$$
\|u\|_{B V_{H}(\Omega)}=\|u\|_{L^{1}(\Omega)}+\operatorname{Var}_{H}(u ; \Omega) .
$$

The notation $B V_{H, l o c}(\Omega)$ indicates the collection of functions $u \in L_{l o c}^{1}(\Omega)$, such that $u \in B V_{H}(\omega)$, for every $\omega \subset \subset \Omega$. We denote with $B V_{H, l o c}^{2}(\Omega)$ the Banach space of functions $u \in \mathcal{L}_{l o c}^{1,1}(\Omega)$ such that $X_{i} u \in B V_{H, l o c}(\Omega), i=1, \ldots, m$.

Theorem 7.7. Let $u$ be weakly $H$-convex in $\Omega \subset \mathbb{H}^{n}, n=1,2$; then $u \in B V_{H, l o c}^{2}(\Omega)$.

Proof. By Theorems 7.2 and 7.6 we know that $u$ is locally Lipschitz in $\Omega$ with respect to the Carnot-Carathéodory metric, and therefore $X_{j} u \in L_{l o c}^{\infty}(\Omega)$. In particular, $X_{j} u \in L_{l o c}^{1}(\Omega), j=1, \ldots, 2 n$. Let $\omega \subset \subset \Omega$, and consider $\zeta \in \mathcal{F}_{H}(\omega)$. For 
any $i=1, \ldots, 2 n$ we have

$$
\begin{aligned}
& \int_{\omega} X_{i} u \sum_{j=1}^{2 n} X_{j} \zeta_{j} d g=-\sum_{j=1}^{2 n} \int_{\omega} u X_{i} X_{j} \zeta_{j} d g \\
& =-2 \sum_{j=1}^{2 n} \int_{\omega} u \frac{X_{i} X_{j} \zeta_{j}+X_{j} X_{i} \zeta_{j}}{2} d g+\sum_{j=1}^{2 n} \int_{\omega} u X_{j} X_{i} \zeta_{j} d g .
\end{aligned}
$$

Using Theorem 7.4 we obtain from (7.10) that

$$
\int_{\omega} X_{i} u \sum_{j=1}^{2 n} X_{j} \zeta_{j} d g=-2 \sum_{j=1}^{2 n} \int_{G} \zeta_{j}(g) d \nu_{H}^{i j}(g)+\sum_{j=1}^{2 n}\left(X_{i} X_{j} u, \zeta_{j}\right),
$$

where we have denoted by $(\cdot, \cdot)$ the duality between $\mathcal{D}^{\prime}(\boldsymbol{G})$ and $\mathcal{D}(\boldsymbol{G})$. By Theorem 7.5 we know that $X_{i} X_{j} u$ are also Radon measures, therefore we conclude

$$
\int_{\omega} X_{i} u \sum_{j=1}^{2 n} X_{j} \zeta_{j} d g \leq 2 \sum_{j=1}^{2 n} \nu_{H}^{i j}(\omega)+\sum_{j=1}^{2 n} X_{i} X_{j} u(\omega)<\infty .
$$

Taking the supremum on all $\zeta \in \mathcal{F}_{H}(\omega)$ we reach the conclusion that for every $i=1, \ldots, 2 n, X_{i} u \in B_{H}(\omega)$, hence $u \in B V_{H}^{2}(\omega)$. This completes the proof.

Theorem 7.7 now allows us to close the gap between Theorem 9.2 from [DGN1] (for a statement of this result see Theorem 8.1 in the next section) and an integral version of the Busemann-Feller-Alexandrov theorem recently established in AM. Since the argument is from this point on a standard modification of that given in the classical case in Theorem 6.4.1 in EG], we omit it and refer the interested reader to [DGNT, or [M]. We thus obtain a sub-elliptic counterpart of the classical theorem of Busemann-Feller and Alexandrov. We recall that the latter states that a convex function admits second derivatives at a.e. point.

Theorem 7.8. Let $u: \Omega \rightarrow \mathbb{R}$ be a weakly $H$-convex function in an $H$-convex open set $\Omega \subset \mathbb{H}^{n}, n=1,2$. For $d g$-a.e. point $g_{o} \in \Omega$ there exists a polynomial of weighted degree $2, P_{u}\left(g ; g_{o}\right)$, such that

$$
\lim _{g \rightarrow g_{o}} \frac{u(g)-P_{u}\left(g ; g_{o}\right)}{d\left(g, g_{o}\right)^{2}}=0 .
$$

In particular, the second derivatives $X_{i} X_{j} u(g), i, j=1, \ldots, 2 n$, exist at dg-a.e. point $g \in \Omega$.

\section{Estimates from Below of the generalized Monge-Ampère measure}

The purpose of this section is to clarify the connection between Theorem 1.1 and the estimate in Theorem 1.2 from GM. As we have mentioned in the Introduction, considering Theorem 1.2 a maximum principle similar to Alexandrov's estimate is not appropriate, since such a result is not a geometric maximum principle (for the geometric maximum principle, see the discussion in the next section). To clarify this point in the present section we give a completely elementary proof of Theorem 1.2. which does not use any of the tools employed in GM], and in particular makes no use of the monotonicity of Theorem 1.1. In fact, for every $\mathbb{H}^{n}$ we prove a stronger statement, Theorem 8.3 , that can be directly deduced from the standard Poincaré 
inequality, and from the following compactness result, which is Theorem 9.2 in DGN1.

Theorem 8.1. In a Carnot group $\boldsymbol{G}$, let $u \in C(\boldsymbol{G})$ be a weakly $H$-convex function. Then there exists $C=C(\boldsymbol{G})>0$ such that for every ball $B(g, r)$ one has

$$
\sup _{B(g, r)}|u| \leq C \frac{1}{|B(g, 5 r)|} \int_{B(g, 5 r)}|u| d g^{\prime}
$$

and

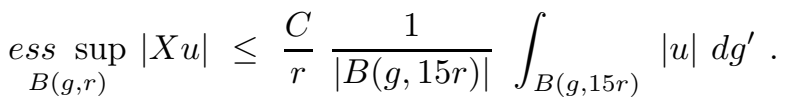

In particular, (8.1) implies that there exist no $L^{1}$, continuous, weakly $H$-convex functions in $\boldsymbol{G}$, other than the trivial one.

We begin with a simple consequence of the classical one-dimensional Poincarétype inequality.

Lemma 8.2. Consider a gauge ball $B(0, R) \subset \mathbb{H}^{n}$. Let

$$
u \in \Gamma^{2}(B(0, R)) \cap C(\bar{B}(0, R)),
$$

with $u=0$ on $\partial B(0, R)$. Then there exists an absolute constant $C>0$ such that

$$
\int_{B(0, R)} u^{2}(g) d g \leq C R^{4} \int_{B(0, R)} T u(g)^{2} d g .
$$

Proof. We first assume that $R=1$. Recall that $B(0,1)=\left\{g=(z, t) \in \mathbb{H}^{n} \mid N(g)<\right.$ $1\}$, where $N(g)=\left(|z|^{4}+16 t^{2}\right)^{1 / 4}$. For every $g=(z, t) \in B(0, R)$, we have

$$
u(z, t)=\int_{-1 / 4}^{t} T u(z, s) d s,
$$

where we have made use of the hypothesis $u=0$ on $\partial B(0,1)$. In what follows we continue to indicate with $u$ the extension of such a function with zero outside $B(0,1)$. The latter equality implies in a standard fashion

$$
\begin{aligned}
& \int_{B(0,1)} u(z, t)^{2} d z d t \leq \frac{1}{2} \int_{B(0,1)} \int_{-1 / 4}^{1 / 4} T u(z, s)^{2} d s d z d t \\
& =\frac{1}{2} \int_{-1 / 4}^{1 / 4} \int_{|z|<\left(1-16 t^{2}\right)^{1 / 4}} \int_{-1 / 4}^{1 / 4} T u(z, s)^{2} d s d z d t \\
& \leq \frac{1}{2} \int_{-1 / 4}^{1 / 4} \int_{-1 / 4}^{1 / 4} \int_{|z|<1} T u(z, s)^{2} d z d s d t=\frac{1}{4} \int_{B(0,1)} T u(z, s)^{2} d z d s .
\end{aligned}
$$

This proves the lemma when $R=1$. The case of general $R$ is now recovered by a simple rescaling argument. If $u$ is as in the statement of the lemma, one considers $u_{R}(z, t)=u\left(R z, R^{2} t\right)$. Keeping in mind that $u_{R}$ lives on $B(0,1)$, and that $T u_{R}(z, t)=R^{2} T u\left(R z, R^{2} t\right)$, from (8.3) one obtains

$$
\int_{B(0,1)} u\left(R z, R^{2} t\right)^{2} d z d t \leq \frac{R^{4}}{4} \int_{B(0,1)} T u\left(R z, R^{2} t\right)^{2} d z d t .
$$

To finish the proof we make the change of variable $\left(z^{\prime}, t^{\prime}\right)=\left(R z, R^{2} t\right)$ in the latter inequality. 
Using Lemma 8.2 and Theorem 8.1 we can now establish a basic estimate from below for the commutator of a weakly $H$-convex function.

Theorem 8.3. Let $B=B\left(g_{o}, R\right) \subset \mathbb{H}^{n}$, and consider a weakly $H$-convex function $u \in \Gamma^{2}(B) \cap C(\bar{B})$ such that $u=0$ on $\partial B$. There exists a geometric constant $\alpha_{n}>0$ such that for every $g \in B\left(g_{o}, R\right)$ one has

$$
|u(g)| \leq \alpha_{n} \frac{R^{n+2-\frac{1}{n}}}{\operatorname{dist}(g, \partial B)^{n+1}}\left(\int_{B}(T u)^{2 n} d g^{\prime}\right)^{\frac{1}{2 n}} .
$$

In particular, we obtain

$$
\max _{B\left(g_{o}, R / 2\right)}|u| \leq \tilde{\alpha}_{n} R^{1-\frac{1}{n}}\left(\int_{B}(T u)^{2 n} d g^{\prime}\right)^{\frac{1}{2 n}} .
$$

Proof. By left-translation we can, without restriction, assume that $g_{o}=0$, the group identity. Let $g \in B$ and denote by $\rho=\operatorname{dist}(g, \partial B)$ the gauge distance of $g$ to $\partial B$. Since Theorem 8.1 has a local character, we can apply the estimate (8.1) to the function $u$ in $B(g, \rho / 10)$, obtaining for some absolute constant $C=C(n)>0$

$$
\begin{aligned}
|u(g)| & \leq \max _{B(g, \rho / 10)}|u| \leq \frac{C}{|B(g, \rho / 2)|} \int_{B(g, \rho / 2)}\left|u\left(g^{\prime}\right)\right| d g^{\prime} \\
& \leq C\left(\frac{1}{\left|B\left(g, \frac{\rho}{2}\right)\right|} \int_{B\left(g, \frac{\rho}{2}\right)}\left|u\left(g^{\prime}\right)\right|^{2} d g^{\prime}\right)^{\frac{1}{2}} \\
& \leq \frac{C}{\left|B\left(g, \frac{\rho}{2}\right)\right|^{\frac{1}{2}}}\left(\int_{B(0, R)} u\left(g^{\prime}\right)^{2} d g^{\prime}\right)^{1 / 2} .
\end{aligned}
$$

We now apply Lemma 8.2 to (8.4), obtaining

$$
\begin{aligned}
|u(g)| & \leq \frac{C R^{2}}{\left|B\left(g, \frac{\rho}{2}\right)\right|^{\frac{1}{2}}}\left(\int_{B(0, R)} T u\left(g^{\prime}\right)^{2} d g^{\prime}\right)^{1 / 2} \\
& \leq \frac{C R^{n+2-\frac{1}{n}}}{\rho^{n+1}}\left(\int_{B(0, R)} T u\left(g^{\prime}\right)^{2 n} d g^{\prime}\right)^{1 / 2 n} .
\end{aligned}
$$

A corollary of Theorem 8.3 is the following estimate from above which generalizes Theorem 1.2. Because of the commutator Tu, such an estimate is a direct consequence of Theorem 8.3, and Theorems 1.1 or 2.1 play no role in its proof, nor does the geometry of the gauge ball in $\mathbb{H}^{n}$.

Corollary 8.4. Let $B=B\left(g_{o}, R\right) \subset \mathbb{H}^{n}, n=1,2$, and consider a weakly $H$-convex function $u \in \Gamma^{2}(B) \cap C(\bar{B})$ such that $u=0$ on $\partial B$. There exists a geometric constant $\alpha_{n}>0$ such that for every $g \in B\left(g_{o}, R\right)$ one has

$$
|u(g)| \leq \alpha_{n} \frac{R^{n+2-\frac{1}{n}}}{\operatorname{dist}(g, \partial B)^{n+1}}\left(\int_{B} \mathcal{S}_{m a}(u)\left(g^{\prime}\right) d g^{\prime}\right)^{\frac{1}{2 n}} .
$$

Proof. The case $n=1$ is completely trivial, since thanks to Theorem 2.2 we have

$$
\mathcal{S}_{m a}(u)=H(u)+\frac{3}{4}(T u)^{2} \geq \frac{3}{4}(T u)^{2},
$$


and therefore the conclusion follows immediately from Theorem 8.3. In the case $n=2$, the proof is the same, except that we now need to resort to Theorem 2.2. and to Theorem 3.2, to conclude that

$$
\mathcal{S}_{m a}(u) \geq \frac{5}{16}(T u)^{4} .
$$

\section{On the Commutator term in Theorem 2.1. AND A BASIC OPEN QUESTION}

In Remark 6.7 we have seen that, because of Proposition 6.6, it is not possible to replace $\mathcal{S}_{m a}(u)$ with $\operatorname{det} \operatorname{Hess}_{X}(u)$ in Corollary 8.4. Here, we want to give this negative phenomenon a broader perspective by connecting it to the best possible character of certain inequalities of $\mathrm{ABP}$ type which presently constitute a fundamental open question to further the development of the theory.

One of the central tools in the theory of equations of Monge-Ampère type is the geometric maximum principle due to Alexandrov-Bakelman-Pucci; see Theorem 9.1 in GT, or also the original papers [A1, A2, Ba1, Ba2, $\mathrm{Pu} 1, \mathrm{Pu} 2$. If one wants to develop a theory of fully nonlinear equations in Carnot groups, it is natural to consider corresponding linear sub-elliptic equations with rough coefficients. For a symmetric, positive definite $m \times m$ matrix-valued function $g \rightarrow A(g)=\left(a_{i j}(g)\right)$ on $\boldsymbol{G}$ with measurable entries, we form the second order nonvariational operator

$$
L u \stackrel{\text { def }}{=} \sum_{i, j=1}^{m} a_{i j} u_{, i j}=\operatorname{trace}\left[\operatorname{AHess}_{X}(u)\right] .
$$

In DGN1 the authors formulated as a conjecture the following a priori estimate:

$$
\sup _{\Omega} u \leq \sup _{\partial \Omega} u^{+}+C\|L u\|_{L^{Q}(\Omega)},
$$

where $Q$ is the homogeneous dimension of $\boldsymbol{G}$ associated with the weighted grading of the Lie algebra. When $a_{i j} \equiv \delta_{i j}$, then $L=\sum_{i=1}^{m} X_{i}^{2} u$ is the sub-Laplacian associated with the system $X=\left\{X_{1}, \ldots, X_{m}\right\}$, and the strong maximum principle for this operator is a special case of the pioneering work of Bony [Bo. In such a situation one can prove that when $F \in L^{p}(\Omega)$ for some $p>Q / 2$, then $u$ belongs to $L^{\infty}(\Omega)$, and one has the estimate

$$
\sup _{\Omega}|u| \leq C(\boldsymbol{G}, p) \operatorname{diam}(\Omega)^{2-Q / p}\|F\|_{L^{p}(\Omega)} .
$$

The $L^{p}$ norm on the right-hand side of (9.3) is best possible, in the sense that it cannot be replaced by the smaller $\|F\|_{L^{Q / 2}(\Omega)}$. To see this it suffices to consider in the Heisenberg group $\mathbb{H}^{n}$ the function $u=\log |\log N|$, where $N(g)=$ $\left(\left(|x|^{2}+|y|^{2}\right)^{2}+16 t^{2}\right)^{1 / 4}$ is the anisotropic gauge [F1]. Using formulas from [FS1] one easily checks that if $\mathcal{L}$ denotes the real part of the Kohn-Spencer sub-Laplacian on $\mathbb{H}^{n}$, then $u$ solves the Dirichlet problem $\mathcal{L} u=F$ with zero boundary conditions in $\Omega=B(0, R)=\left\{g \in \mathbb{H}^{n} \mid N(g)<R\right\}$, where $0=(0,0,0)$ is the group identity, $R=e^{-1}$, and $F=O\left(\frac{1}{N^{2}|\log N|}\right)$ in $\Omega$. By the polar coordinates in [FS1], [FS2] one also recognizes that $F \in L^{Q / 2}(\Omega)$. Since on the other hand $u \notin L^{\infty}(\Omega)$, it is clear that for such an example (9.3) cannot possibly hold with $p=Q / 2$. 
What happens when $a_{i j} \not \equiv \delta_{i j}$ in (9.1)? From the classical geometric-arithmetic mean inequality one has

$$
\operatorname{det} \operatorname{Hess}_{X}(u) \leq \frac{1}{m^{m}} \frac{\left[\sum_{i, j=1}^{m} a_{i j} u_{, i j}\right]^{m}}{\operatorname{det}\left(a_{i j}\right)} .
$$

This shows that, if for functions $u \in \Gamma^{2}(\Omega) \cap C(\bar{\Omega})$ with $u=0$ on $\partial \Omega$ one could establish a control of the type

$$
\sup _{\Omega}|u| \leq C\left(\int_{\Omega}\left|\operatorname{det} \operatorname{Hess}_{X}(u)\right| d g\right)^{1 / m},
$$

with $C=C(\Omega)>0$, then for a solution $u \in \Gamma^{2}(\Omega) \cap C(\bar{\Omega})$ of $L u=F$ in $\Omega$, vanishing on $\partial \Omega$, one would obtain the following estimate:

$$
\sup _{\Omega}|u| \leq C\left\|\frac{F}{\left(\operatorname{det}\left(a_{i j}\right)\right)^{1 / m}}\right\|_{L^{m}(\Omega)} .
$$

The estimate (9.4) appears as the natural sub-Riemannian analogue of the geometric lemma (1.9), which is at the heart of the proof of the ABP maximum principle. We emphasize that, at least for the Heisenberg group $\mathbb{H}^{n}$, with $n \geq 2$, the ensuing estimate (9.5) would not be in contrast with the mentioned optimality of (9.3) since in this setting we have $Q=2 n+2$, whereas $m=2 n$, and therefore $m>Q / 2=n+1$ is always true. It seems thus natural that (9.4) has been conjectured by several people as the appropriate sub-elliptic version of the ABP estimate (1.9). However, in the recent paper [DGN2] the authors have proved the following surprising result.

Theorem 9.1. Let $\boldsymbol{G}$ be a group of Heisenberg type, with homogeneous dimension $Q$, and denote by $\Omega$ the gauge ball $B(0,1)$. For every $0<\epsilon<Q$ there exists a (real) matrix-valued function $A^{\epsilon}(g)=\left(a_{i j}^{\epsilon}(g)\right)$ with symmetric and bounded measurable entries, and satisfying for some $\nu_{\epsilon}>0$ and for a.e. $g \in \boldsymbol{G}$ the uniform ellipticity assumption

$$
\nu_{\epsilon}|\zeta|^{2} \leq \sum_{i, j=1}^{m} a_{i j}^{\epsilon}(g) \zeta_{i} \zeta_{j} \leq \nu_{\epsilon}^{-1}|\zeta|^{2}, \quad \zeta \in \mathbb{R}^{m},
$$

such that the Dirichlet problem

$$
\left\{\begin{array}{l}
L^{\epsilon} u=\sum_{i, j=1}^{m} a_{i j}^{\epsilon} u_{, i j}=0 \quad \text { in } \Omega, \\
u=0 \quad \text { on } \partial \Omega,
\end{array}\right.
$$

admits a solution $u=u^{\epsilon} \in \mathcal{L}^{2, Q-\epsilon} \cap C(\bar{\Omega})$ different from the trivial one.

As a consequence of Theorem 9.1 we see that an estimate such as (9.5) cannot possibly hold since, if it did, it would imply the uniqueness in the Dirichlet problem in the functional class $\mathcal{L}^{2, m}(\Omega)$. But such uniqueness fails, as one can see by taking $\epsilon=Q-m>0$ in Theorem 9.1. From this fact and from the above considerations, we infer that the ABP-type estimate (9.4) cannot possibly hold either.

Summarizing, if we insist on controlling the supremum of $u$ by an $L^{p}$ norm of $L u$, where $L$ ranges in the class of operators of the type (9.1), then Theorem 9.1 says that the smallest allowable $p$ is $Q$, i.e., an estimate such as (9.2). The conjectured a priori inequality (9.2) presently constitutes a basic open problem. 


\section{ACKNOWLEDGMENTS}

We thank N. Krylov for enlightening conversations over several years, L. Capogna, D. Danielli, H. Donnelly and D.M. Nhieu for helpful discussions (in particular, D.M. Nhieu also helped with the proof of Lemma 3.1), C. Gutierrez for his mentioned comments on the role of the commutator in the monotonicity inequality in Theorem 1.1, and N. Trudinger for informing us about [TW] and kindly sharing with us his notes on $k$-Hessian measures. This paper crucially rests on the results in DGN1, DGN2, and could not have been written without these contributions.

\section{REFERENCES}

[A1] A. D. Alexandrov, Certain estimates for the Dirichlet problem, Soviet Math. Dokl., 1 (1960), 1151-1154. MR0147776 (26:5290)

[A2] The impossibility of general estimates for solutions and of uniqueness conditions for linear equations with norms weaker than $L^{n}$, Vestnik Leningrad. Univ., 21 (1966), (13), 5-10. MR0197952(33:6111)

[AM] L. Ambrosio \& V. Magnani, Weak differentiability of BV functions on stratified groups, Math. Zeit., 245 (2003), no. 1, 123-153. MR2023957 (2005a:43010)

[Ar1] G. Aronsson, Extension of functions satisfying Lipschitz conditions, Ark. Mat., 6 (1967), 551-561. MR0217665 (36:754)

[Ar2] On the partial differential equation $u_{x}{ }^{2} u_{x x}+2 u_{x} u_{y} u_{x y}+u_{y}{ }^{2} u_{y y}=0$, Ark. Mat., 7 (1968), 395-425. MR0237962 (38:6239)

[Ba1] I. Ja. Bakelman, Theory of quasilinear elliptic equations, Sibirsk. Mat. Ž. (in Russian), 2 (1961), 179-186. MR0126604 (23:A3900)

[Ba2] , Geometric methods for solving elliptic equations, "Nauka", Moscow, 1965. MR0194727 (33:2933)

[Ba3] Convex Analysis and Nonlinear Geometric Elliptic Equations, Springer-Verlag, 1994. MR 1305147 (95k:35063)

[BR] Z. M. Balogh \& M. Rickly, Regularity of convex functions on Heisenberg groups, Ann. Sc. Norm. Super. Pisa Cl. Sci., 5, 2 (2003), no. 4, 847-868. MR2040646

[Be] A. Bellaïche \& J.-J. Risler, ed., Sub-Riemannian Geometry, Birkhäuser, 1996. MR.1421821 (97f:53002)

[B] T. Bieske, On $\infty$-harmonic functions on the Heisenberg group, Comm. PDE, no.3\&4, 27 (2002), 727-761. MR 1900561 (2003g:35033)

[BC] T. Bieske \& L. Capogna, The Aronsson-Euler equation for absolutely minimizing Lipschitz extensions with respect to Carnot-Carathéodory metrics, Trans. Amer. Math. Soc. 357 (2005), no. 2, 795-823. MR2095631 (2005g:35036)

[Bo] J. M. Bony, Principe du maximum, inégalité de Harnack et unicité du problème de Cauchy pour les operateurs elliptique degeneres, Ann. Inst. Fourier, Grenoble, 1, 119 (1969), 277-304. MR0262881 (41:7486)

[CC] L. A. Caffarelli \& X. Cabré, Fully Nonlinear Elliptic Equations, Amer. Math. Soc., Colloquium Publ., vol. 43, 1991. MR.1351007 (96h:35046)

[CDG] L. Capogna, D. Danielli \& N. Garofalo, The geometric Sobolev embedding for vector fields and the isoperimetric inequality, Comm. Anal. and Geom., 2 (1994), 201-215. MR1312686 (96d:46032)

[CGr] L. Corwin \& F. P. Greenleaf, Representations of nilpotent Lie groups and their applications, Part I: basic theory and examples, Cambridge Studies in Advanced Mathematics 18, Cambridge University Press, Cambridge (1990). MR.1070979 (92b:22007)

[CDKR] M. Cowling, A. H. Dooley, A. Korányi \& F. Ricci, H-type groups and Iwasawa decompositions, Adv. Math., 87 (1991), 1-41. MR1102963 (92e:22017)

[CIL] M. Crandall, H. Ishii \& P. L. Lions, User's guide to viscosity solutions of second order partial differential equations, Bull. Amer. Math. Soc., 27 (1992), no.1, 1-67. MR1118699 $(92 \mathrm{j}: 35050)$

[Cy] J. Cygan, Subadditivity of homogeneous norms on certain nilpotent Lie groups, Proc. Amer. Math. Soc., 83 (1981), 69-70. MR0619983(82k:22009) 
[Da] B. Dacorogna, Direct Methods in the Calculus of Variations, Springer-Verlag, 1989. MR0990890 (90e:49001)

[DGN1] D. Danielli, N. Garofalo \& D. M. Nhieu, Notions of convexity in Carnot groups, Comm. Analysis and Geometry, 11, no.2, (2003), 263-341. MR2014879 (2004m:22014)

[DGN2] - On the best possible character of the $L^{Q}$ norm in some a priori estimates for non-divergence form equations in Carnot groups, Proc. Amer. Math. Soc., 131, no.11, (2003), 3487-3498. MR1991760 (2004i:35051)

[DGN3] — Minimal surfaces in Carnot groups, preprint, 2003.

[DGNT] D. Danielli, N. Garofalo, D. M. Nhieu \& F. Tournier, The theorem of Busemann-FellerAlexandrov for convex functions in Carnot groups, Comm. Analysis and Geometry, 12 (2004), no. 4, 853-886. MR2104079 (2005j:22005)

[E] L. C. Evans, Partial Differential Equations, Grad. Studies in Math., vol.19, Amer. Math. Soc., 1998. MR.1625845 (99e:35001)

[EG] L. C. Evans \& R. F. Gariepy, Measure Theory and Fine Properties of Functions, CRC Press, 1992. MR1158660 (93f:28001)

[F1] G. B. Folland, A fundamental solution for a sub-elliptic operator, Bull. Amer. Math. Soc., 79 (1973), 373-376. MR0315267 (47:3816)

[F2] Sub-elliptic estimates and function spaces on nilpotent Lie groups, Ark. Math., 13 (1975), 161-207. MR0494315 (58:13215)

[FS1] G. B. Folland \& E. M. Stein, Estimates for the $\bar{\partial}_{b}$ complex and analysis on the Heisenberg group, Comm. Pure Appl. Math., 27 (1974), 429-522. MR0367477 (51:3719)

[FS2] , Hardy Spaces on Homogeneous Groups, Princeton Univ. Press, 1982. MR0657581 (84h:43027)

[G] N. Garofalo, Analysis and Geometry of Carnot-Carathéodory Spaces, With Applications to Pde's, Birkhäuser, book in preparation.

[GN] N. Garofalo \& D. M. Nhieu, Isoperimetric and Sobolev inequalities for CarnotCarathéodory spaces and the existence of minimal surfaces, Comm. Pure Appl. Math., 49 (1996), 1081-1144. MR1404326 (97i:58032)

[GT] D. Gilbarg \& N. Trudinger, Elliptic Partial Differential Equations of Second Order, 2nd ed., Grundleheren der Mathematischen Wissenschaften, 224, Springer, 1998. MR0737190 (86c:35035)

[Gu] C. E. Gutierrez, The Monge-Ampère Equation, Birkhäuser, 2001. MR:1829162 (2002e:35075)

[GM] C. E. Gutierrez \& A. Montanari, Maximum and comparison principles for convex functions on the Heisenberg group, preprint, first posted on May 31, 2003, to the webpage www.dm.unibo.it/ montanar

[H] H. Hörmander, Hypoelliptic second-order differential equations, Acta Math., 119 (1967), 147-171. MR0222474 (36:5526)

[Hou] A. S. Householder, The theory of matrices in numerical analysis, Blaisdell Publishing Co. Ginn and Co., New York-Toronto-London, 1964. MR0175290 (30:5475)

[J] R. Jensen, Uniqueness of Lipschitz extensions: minimizing the sup norm of the gradient, Arch. Rational Mech. Anal., 123 (1993), no. 1, 51-74. MR.1218686 (94g:35063)

[Ka] A. Kaplan, Fundamental solutions for a class of hypoelliptic PDE generated by composition of quadratic forms, Trans. Amer. Math. Soc., 258 (1980), 147-153. MR0554324 (81c:58059)

[K] N. V. Krylov, Sequences of convex functions, and estimates of the maximum of the solution of a parabolic equation, Sibirski Math. Zh., no.2, 17 (1976), 226-236. MR0420016 $(54: 8033)$

[LMS] G. Lu, J. Manfredi \& B. Stroffolini, Convex functions on the Heisenberg group, Calc. Variat. \& Part. Diff. Eq., 19 (2004), no. 1, 1-22. MR2027845 (2004m:35088)

[M] V. Magnani, Lipschitz continuity, Alexandrov theorem, and characterizations for $H$ convex functions, preprint, September 2003.

[Mon] R. Montgomery, A Tour of Subriemannian Geometries, Their Geodesics and Applications, Mathematical Surveys and Monographs, 91. American Mathematical Society, Providence, RI, 2002. MR1867362 (2002m:53045)

[P] P. Pansu, Métriques de Carnot-Carathéodory et quasi-isométries des espaces symétriques de rang un, Ann. of Math., (2)129 (1989), no. 1, 1-60. MR0979599 (90e:53058) 
[Pu1] C. Pucci, Operatori ellittici estremanti, Ann. Mat. Pura Appl., (4) 72 (1966), 141-170. MR0208150 (34:7960)

[Pu2] Limitazioni per soluzioni di equazioni ellittiche, Ann. Mat. Pura Appl., (4) 74 (1966), 15-30. MR.0214905 (35:5752)

[S] E. M. Stein, Harmonic Analysis: Real Variable Methods, Orthogonality and Oscillatory Integrals, Princeton Univ. Press, 1993. MR.1232192 (95c:42002)

[Sto1] S. Stojanovic, Computational Financial Mathematics using Mathematica: optimal trading in stocks and options, Birkhäuser, 2002. MR1930223(2003i:91056)

[Sto2] - Optimal momentum edging via hypoelliptic reduced Monge-Ampère PDE's, preprint, 2003.

[TW] N. S. Trudinger \& X. J. Wang, Hessian measures, Top. Methods in Nonlin. Anal., 10 (1997), 225-239. MR.1634570 (2000a:35061)

[V] V. S. Varadarajan, Lie Groups, Lie Algebras, and Their Representations, SpringerVerlag, New York, Berlin, Heidelberg, Tokyo, 1974. MR0376938 (51:13113)

[Wa1] C. Wang, The Aronsson equation for absolute minimizers of $L^{\infty}$ functionals associated with vector fields satisfying Hörmander's condition, preprint, May 2003.

[Wa2] , The comparison principle for viscosity solutions of fully nonlinear sub-elliptic equations in Carnot groups, preprint, July 2003.

[Wa3] - Viscosity convex functions on Carnot groups, Proc. Amer. Math. Soc., 133 (2005), no. 4, 1247-1253. MR2117228(2006a:58026)

Department of Mathematics, Purdue University, West Lafayette, Indiana 47907

E-mail address: garofalo@math.purdue.edu

Department of Mathematics, Purdue University, West Lafayette, Indiana 47907

E-mail address: tournier@math.purdue.edu

E-mail address: fedeleti@aim.com 